\title{
Combining experimental and theoretical evidence to understand predator learning behaviour with unfamiliar prey
}

\author{
by \\ Lauren Kelly Efford
}

A thesis submitted to the Faculty of Graduate and Postdoctoral Affairs in partial fulfillment of the requirements for the degree of

\section{Master of Science}

in

Biology

Carleton University

Ottawa, Ontario

(C) 2019

Lauren Efford 


\begin{abstract}
Predators confronted with an unfamiliar prey must decide whether or not to attack it. This decision is dependent on the predator's previous experiences with the prey type. In this thesis, I begin by testing the ability of Jamaican field crickets, Gryllus assimilis, to learn a novel binary food choice between a rewarding and unrewarding prey using visual discriminatory cues. In this experiment, evidence of learning was confirmed across trials. Moreover, the colour of the prey item significantly affected the probability of crickets choosing the palatable option, with palatable green prey more likely to be attacked than palatable blue prey. I then developed a model that formalized prey selection in terms of an exploration-exploitation trade-off. With this model, I identified the optimal sampling strategy for a predator with Bayesian learning. I demonstrate that a predator's prior beliefs (Bayesian priors), and the certainty it has in its beliefs (variance in prior) affect the optimal sampling strategy, and hence the nature of selection it places on prey.
\end{abstract}




\section{Acknowledgments}

I would first like to extend my most sincere gratitude and thanks to my co-supervisors Drs. Tom Sherratt and Jean-Guy Godin for their continual support and guidance throughout my Master's degree. I am truly thankful for the irreplaceable opportunity you both have given me, and for all of the experiences and lessons that have come with it. I would also like to express many thanks to the members of my thesis advisory committee, Drs. Sue Bertram and Julie Morand-Ferron, for their instrumental feedback and input throughout this process. As well, a special thank you goes to Dr. Bertram for "adopting" me as an honorary member of her lab, and for her endless generosity and support as I completed my research.

Thank you to the many members of the Sherratt, Godin, and Bertram labs who have extended their support and kindness. A special thank you to Vandana Gopeechund and Peter Sourges for being tremendous lab and field assistants, and to Sophie Potter for her endless generosity, guidance, and friendship.

Lastly, my greatest appreciation and deepest thanks goes to my family; my parents Jim and Kelly Efford, my brother Colin, and my sister Taylor; and to my partner Travis Douglas for their never-ending support and unconditional love. To my parents, I cannot thank you enough for everything you do, your encouragement, love, and support is overwhelming. To my partner Travis, thank you for always being by my side, and for always knowing how to make me smile. I am lucky to have such a special person in my life. 


\section{Table of Contents}

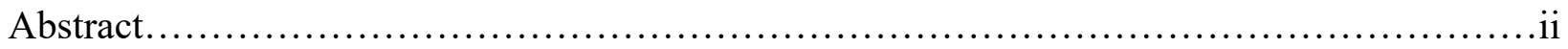

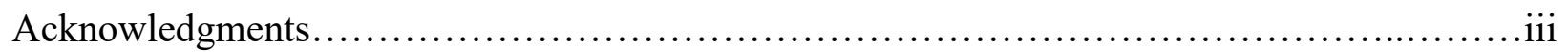

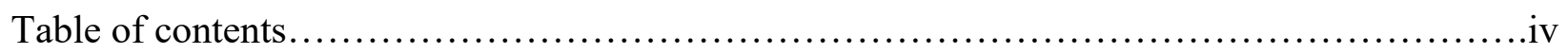

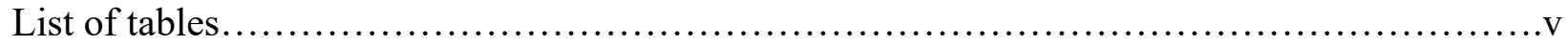

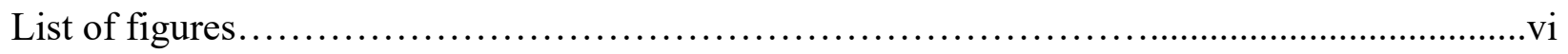

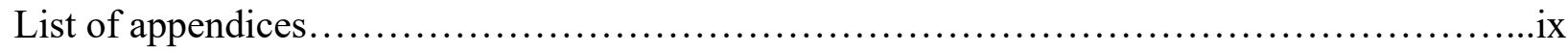

CHAPTER 1. General Introduction.......................................................

CHAPTER 2. Investigating learning and prey selection in Jamaican field crickets............11

CHAPTER 3. Solving an optimal sampling strategy for a predator assuming Bayesian

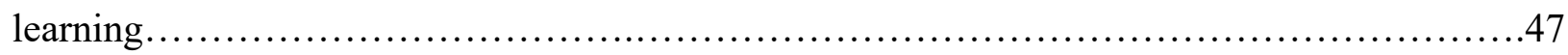

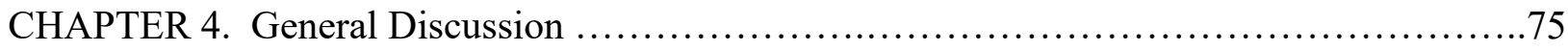

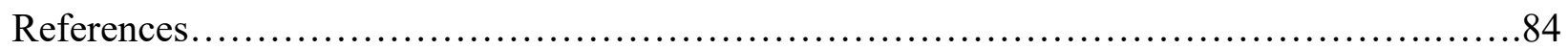

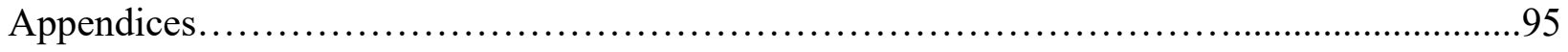




\section{List of Tables}

Table 2.1 Parameter estimates from fitting a generalized linear mixed-effects model testing for the effects of sequence, trial, colour, and sex on the probability of crickets learning a food preference. Shown are the model's parameters, parameter estimates, standard error values, Z values, and $p$-values for each variable. Statistically significant $p$-values $(p \leq 0.05)$ are boldfaced.

Table 2.2 ANOVA results from a generalized linear mixed-effects model testing for the effects of sequence, trial, colour, and sex on the probability of crickets learning a food preference in Trial 5 and the Test trial. Shown are the model's parameters, parameter estimates, standard error values, $\mathrm{Z}$ values, and $\mathrm{p}$-values for each variable. Statistically significant $\mathrm{p}$-values $(\mathrm{p} \leq 0.05)$ are

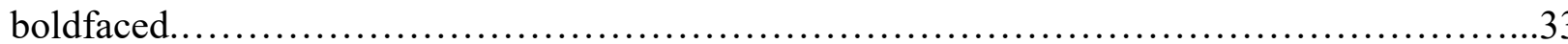

Table 2.3 Replicated goodness-of-fit tests for Trial 1, Trial 5, and the Test Trial. Shown is the individual G statistic, the total G statistic, the pooled G statistic, degrees of freedom, the observed number of correct and incorrect choices made, and p-value for each test. p-values were adjusted for multiple testing using the Bonferroni method $\left(\alpha_{\text {adjusted }}=0.05 / 9\right)=0.0055$.

Statistically significant $\mathrm{p}$-values are shown in bold $\left(\mathrm{p} \leq \alpha_{\text {adjusted }}\right)$ are boldfaced..................36 


\section{List of Figures}

Figure 1.1 Beta distributions for the prior probability distribution (Beta $(1,1)$ ) with expectation 0.5 (red line), and updated posterior probability distribution $(\operatorname{Beta}(8,12))$ with expectation 0.66 (black line).

Figure 2.1 Experimental arena $(32 \times 24 \times 21 \mathrm{~cm}, \mathrm{~L} \times \mathrm{W} \times \mathrm{H})$ constructed from white corrugated plastic. Interior walls were distinguishable by patterns of black duct tape; including horizontal stripes, vertical stripes, a V and a cross. The arena included a total of 14 binary choice stations, each consisting of a green square and blue circle. An acclimatization cup in the center of the arena temporarily held a test cricket. A zone of acclimatization was represented by the area under the cup, outlined above by the red circle.

Figure 2.2 Schematic of experimental set up. Acoustic chamber measuring $121.92 \mathrm{~cm} \times 121.92$ $\mathrm{cm} \times 121.92 \mathrm{~cm}$ and lit by two LED lights were mounted on opposite facing peripheral walls. Cricket behaviour was recorded using a Logitech HD Pro Webcam (model C920) mounted overhead.

Figure 2.3 Mean proportion of rewarding choices made by adult Jamaican field crickets across 5 consecutive learning trials. A) Mean proportion of rewarding prey attacked for all crickets tested. B) Mean proportion of rewarding prey attacked for crickets trained with the rewarding prey coloured green versus blue. Error bars represent standard error of the mean. 
Figure 2.4 Mean proportion of rewarding choices made by adult Jamaican field crickets at each encountered binary choice, spanning from the first to the last binary choice encountered. A) Mean proportion of all 28 crickets across all 5 learning trials. B) Mean proportion of rewarding prey attacked for crickets trained with rewarding prey coloured green versus crickets trained with rewarding prey coloured blue across all 5 learning trials. Error bars represent standard error of the mean.

Figure 2.5 Proportion of "rewarding" choices made by all crickets at each binary choice station encountered. Comparison made between Trial 5 and the Test trial. Error bars represent standard

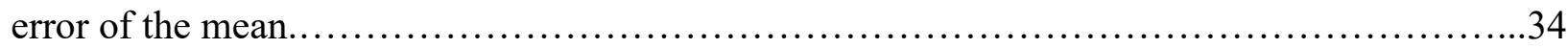

Figure 2.6 Proportion of rewarding choices made by all crickets at each binary choice station encountered. Proportions calculated for each station where a minimum of $50 \%$ of crickets encountered it. Comparison made between Trial 1, Trial 2, Trial 3, Trial 4, Trial 5 and Trial 6 representing the Test trial. Error bars represent standard error of the mean

Figure 3.1 The optimal sampling strategy for a predator assuming Bayesian learning, with set Bayesian priors ( $\alpha$ and $\beta$ ) and mean expectation $(\mu)$ concerning the palatability of 14 unfamiliar prey. 
Figure 3.2 The critical number of unpalatable prey consumed by a predator as a function of the predator's Bayesian prior expectation $(\alpha)$ and variance $\left(\sigma^{2}\right)$ that the prey are unpalatable. The cost of attacking an unrewarding prey was set at 1 , and the benefit of attacking a rewarding prey was set at 2 in the model.

Figure 3.3 Comparison of the optimal sampling strategy to empirical learning data in making a novel binary food choice. Here the optimal sampling strategy follows the assumption that a predator chooses a prey type at random, or a probability of 0.5 . The empirical data is taken from Trial 1 of my previous experiment using Jamaican field crickets.

Figure 3.4 Comparison of the optimal sampling strategy to empirical learning data in making a novel binary food choice. Here the optimal sampling strategy follows the assumption that a predator chooses a prey type based from its prior expectation (Bayesian priors). The empirical data is taken from Trial 1 of my previous experiment using Jamaican field crickets 


\section{List of Appendices}

\section{Appendix A}

An annotated version of the $\mathrm{R}$ code used to develop my Bayesian learning model and to simulate a predator adopting Bayesian learning to respond and learn a novel binary food choice.

Figure A1. Optimal sampling strategy of a predator faced with a sequence of 14 binary food choices consisting of a familiar (palatable) and unfamiliar prey type. The predator begins with an expectation of $0.5(\alpha=1$ and $\beta=1)$ that unfamiliar prey are palatable. I assumed that the predator

uses Bayesian learning to make prey-choice decisions. 


\section{CHAPTER 1}

General Introduction 
The focus of this thesis is to investigate predator learning behaviour using both laboratory experimental and theoretical approaches. Specifically, the thesis aims to elucidate how a predator learns to make a binary choice between two simultaneously available novel food items. This General Introduction is divided into four sections. The first section reviews learning in the context of prey selection. The second is a general overview of Bayesian learning and its application to prey selection. The third section summarizes the goals and objectives of this thesis. The last section outlines the rationale for this study.

\section{Learning}

Learning is a durable and usually adaptive change in an animal's behaviour traceable to a specific experience in the individual's life (Alcock, 2013). Our understanding of learning behaviour has gradually advanced since the $20^{\text {th }}$ century (Dukas, 1998; Shettleworth, 2001; Pearce, 2013). Initially focusing exclusively on vertebrate species, mainly mammals, birds, and fishes, it wasn't until recently that researchers started to investigate learning in invertebrates (Alloway, 1972; Dukas, 2008; Papaj \& Lewis, 2012). The initial lack of interest in invertebrate, and specifically insect, learning has been said to be due to the belief that insects should show little to no learning ability because of their small body and brain sizes, and short lifespan (Mayr, 1974; Alexander et al., 1997). However, research efforts have repeatedly proved these assumptions wrong, and today several insect taxa have even been established as model systems for research on insect learning, including the honey bee (Apis mellifera), parasitoid wasps (e.g. Doryctobracon areolatus), and fruit flies (e.g. Drosophila melanogaster) (e.g. Menzel et al.,1974; Vet et al., 1995; Davis, 2005). 
Recent research indicates that a variety of invertebrate species rely considerably on learning for nearly all of life's major events, such as spatial navigation, predator avoidance, sexual behaviour, social interactions, and prey selection/foraging (Dukas, 2008; Papaj \& Lewis, 2012). For example, spatial learning and homing behaviour have been investigated in several insect species, such as the honeybee (Apis mellifera) who possess the ability to learn the location of a significant landmark (e.g. hive, foraging site etc.) based on personal interactions with their environment (Capaldi et al., 2000). In addition, insects have also been shown to learn in the context of sexual behaviour. For example, Dukas (2005a) demonstrated an effect of learning on mate choice in female fruit flies (Drosophila melanogaster), showing that young virgin females can learn a mate preference based on prior personal experience. Moreover, invertebrate learning has also been tested in the context of predator avoidance. For example, Rochette et al. (1997) demonstrated learned predator recognition and avoidance behaviour by the whelk Buccinum undatum in response to the predatory starfish Leptasterias polaris. Lastly, concentrating specifically on learning in the context of prey selection, invertebrates, ranging from molluscs,

cephalopods, and nematodes, to insect species such as fruit flies and honey bees, have been shown to learn food preferences and avoidances by associating visual or olfactory cues with palatable and unpalatable prey/food sources (Sahley et al., 1980; Darmaillcq et al., 2004; Zhang et al., 2005; Wright et al., 2010; Lee et al., 2018).

\section{Bayesian inference}

Bayesian statistics is based on Bayes' theorem (Ellison, 1996; McNamara \& Houston, 1980), formulated by Thomas Bayes in the late 1700's. In probability statistics, Bayes' theorem describes a degree of belief, or the probability, of an event occurring (hypothesis) based on prior 
knowledge. In Bayesian inference, Bayes' theorem is used to update the probability of an event occurring as more evidence becomes available, consequently computing the posterior probability. Following Bayes and Laplace, it is readily shown that:

$$
P(H \mid E)=\frac{P(E \mid H) P(H)}{P(E)}
$$

Bayes' theorem shows how new knowledge, acquired through individual experiences with their environment, can be incorporated with these prior beliefs to produce a better informed set of posterior beliefs. Bayes’ formula follows immediately from the definition of conditional probability, and can be stated in terms of two components $H$ and $E$, which can be considered a specific Hypothesis (or Hypothesis distribution) and Evidence (data), respectively. Here $P(H \mid E)$ is the posterior probability of the hypothesis $H$ being true given the observed data $E, P(E \mid H)$ is the probability of the data arising if the specific hypothesis were true (i.e. the likelihood), and $P(H)$ is the unconditional prior probability that the specific hypothesis is true. The denominator $P(E)$ represents the unconditional probability of the observed data arising and is equivalent to the sum of the likelihoods of each and every competing hypothesis, multiplied by the prior probability that it is true. $P(E)$ is therefore a normalizing constant, representing the same product as the numerator but here summed (or integrated for hypotheses based on continuous distributions) over all possible hypotheses.

Individual animals continuously make behavioural decisions so as to maximize their fitness. To make these decisions, animals will commonly combine their recent observations and experiences with their prior beliefs. This initial belief is then updated as the animal gathers 
evidence that either confirms or refutes their original bias. This kind of behavioural decision making occurs in a variety of species, and has been repeatedly incorporated into models of animal decision making (e.g. McNamara \& Houston, 1980; Mangel, 1990; Luttbeg, 1996; Olsson \& Holmgren, 1998; Koops \& Abrahams, 2003). Most evidence of animals behaving in a manner consistent with Bayesian inference comes from birds exploiting food patches and estimating food patch quality (Valone \& Brown, 1989; Valone, 1991; Alonso et al., 1995; Olsson et al., 1999; van Gils et al., 2003). For example, van Gils et al. (2003) examined the food patch exploitation behaviour of captive red knots (Calidris canutus) in nearly 50 patches, and found that a Bayesian model best described the patch exploitation behaviour of the birds. Furthermore, this kind of behavioural decision making has also been demonstrated in the context of sexual behaviour and mating decisions (Valone, 2006). For example, Luttbeg and Warner (1999) examined reproductive decision making in peacock wrasse (Symphodus tinaca), and found that the data from the free-ranging wrasse best matched the estimated mating behaviour predicted by a Bayesian model. This model incorporated a prior probability distribution of a female successfully finding a nesting male each day, and assumed that this distribution would be updated according to the number of successful and unsuccessful searches a female experienced each day.

To further illustrate the concept of Bayesian updating, let us consider a situation where a predator encounters an unfamiliar prey and must decide whether or not to attack it. Here, if the outcome of this decision is binomial (for example, the prey is good or bad with a given probability), we will assume both the prior, and hence the posterior, probabilities follow a beta distribution. A beta distribution is a probability distribution ranging from 0 to 1 , defined by two 
shape parameters $\alpha$ and $\beta$. These two parameters establish the shape of the probability distribution, together summarizing the predator's beliefs. An individual will start with a prior probability function defined by $\alpha$ and $\beta$, where the mean expectation is given by $\frac{\alpha}{\alpha+\beta}$. As that individual gains evidence/information to confirm or refute its expectation, the prior probability distribution will be updated to a posterior probability distribution given by $\frac{r+\alpha}{n+\alpha+\beta}$ where $r$ and $n$ are the number of events and trials, respectively. If we think of our predator faced with the decision to attack or defer attack on unfamiliar prey, we can think of $(n)$ as the total number of unfamiliar prey it sampled, and $(r)$ as the number of unfamiliar prey sampled that were unpalatable. Here, let us assume that the predator begins with the prior expectation of 0.5 that all unfamiliar prey are unpalatable, and set $\alpha=1$ and $\beta=1$ (a uniform prior). Following a Bayesian approach, if the predator samples 10 unfamiliar prey and 7 turn out to be unpalatable, then the predator's posterior probability distribution will be updated and now the predator will have an expectation of $(7+1) /(10+1+1)=0.66$ that unfamiliar prey are unpalatable. This relationship is illustrated in Figure 1.1, showing both the predator's prior probability distribution, as well as its updated posterior probability distribution. 


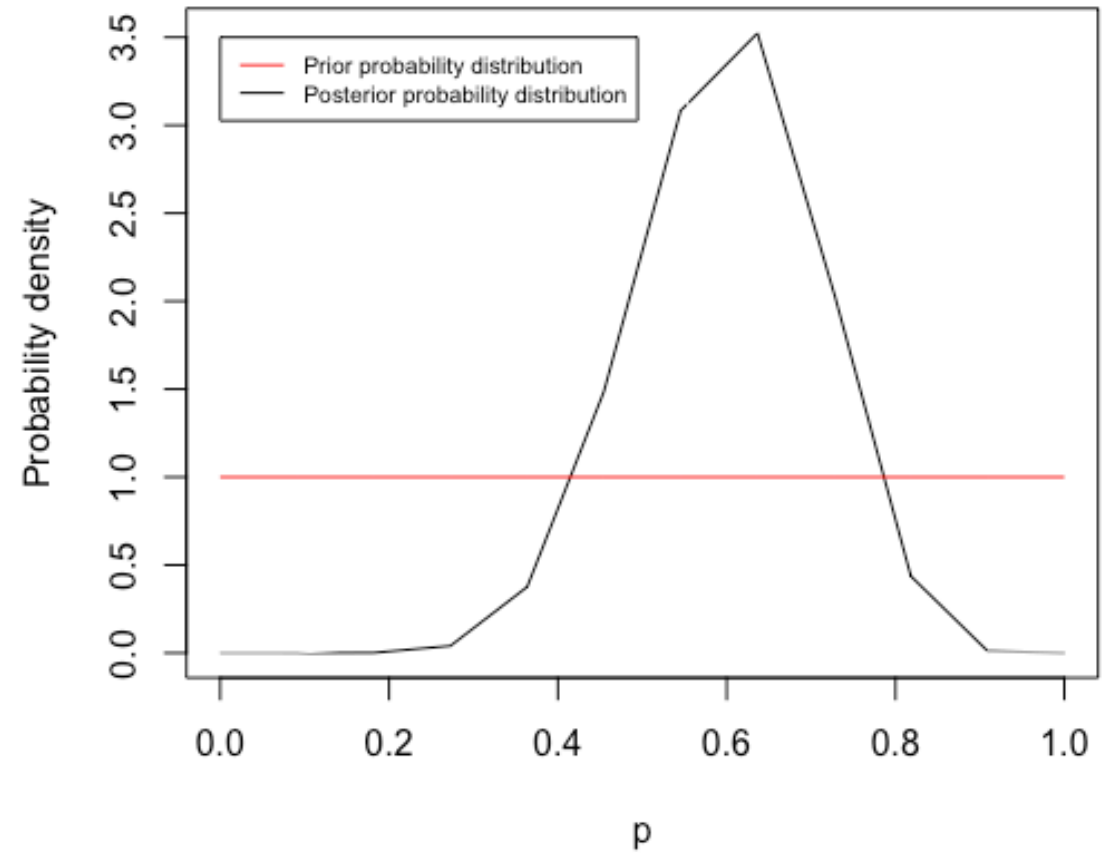

Figure 1.1 Beta distributions for the prior probability distribution (Beta $(1,1))$ with expectation 0.5 (red line), and updated posterior probability distribution $(\operatorname{Beta}(8,12))$ with expectation 0.66 (black line). 


\section{Thesis goals and objectives}

The general goal of my thesis research was to investigate learning in the context of prey selection; more specifically, the learning and foraging behaviour of predators faced with a novel binary food choice. Predators across a broad range of vertebrate taxa have demonstrated the ability to successfully respond to a binary food choice (e.g., MacKay 1974, 1977; Czaplicki et al., 1976; Little, 1977; Mason and Reidinger, 1983; Manteifel \& Karelina, 1996). In addition, evidence of this behaviour has also been observed in several invertebrate species (e.g., Sahley et al.,1980; Dukas, 1999; Darmaillcq et al., 2004; Zhang et al., 2005). For example, Dukas (1999) showed that groups of fruit fly larvae (Drosophila melanogaster) successfully learned to make a binary food choice between a high-quality food consisting of a high protein and carbohydrate content, and a low-quality food lacking all proteins and carbohydrates. Likewise, using a similar binary choice design, Lee et al. (2018) demonstrated that fruit flies (Drosophila melanogaster) will learn to successfully respond to a binary food choice, when presented a choice between a sucrose solution and a solution consisting of a high calcium concentration. In my thesis, my primary aim was to test whether Jamaican field crickets (Gryllus assimilis) are capable of learning a binary food choice, and to model this problem and solve for the optimal sampling strategy.

My thesis is comprised of two chapters: a food choice experiment using Jamaican field crickets, and a theoretical model formalizing this problem as an exploration-exploitation tradeoff and solving for the optimal sampling strategy. First, I conducted a binary food choice experiment to test the ability of individual Jamaican field crickets to learn a food preference using visual discriminatory cues. I used two prey types, a novel rewarding prey (Gala apple) and 
a novel unrewarding prey (fun foam), and dyed both prey either blue or green. Crickets completed 5 days of experimental trials, and each trial consisted of 14 binary food choices. I predicted that crickets would learn a dietary preference by associating the positive stimulus (rewarding prey) and negative stimulus (unrewarding prey) with the corresponding colour of the prey item.

Second, I used a mathematical model to simulate the above-mentioned binary food choice experiment and to solve for the optimal sampling strategy. I formalized this problem as an exploration-exploitation trade-off and solved for the optimal stopping point and the critical number of unpalatable prey a predator should consume before complete avoidance, for a predator assuming Bayesian learning. The primary goal of developing this model was to identify how Bayesian priors influence prey selection and learning, and whether the variance around the predator's mean expectation also influences its decision to attack and whether to keep attacking an unfamiliar prey.

\section{Rationale}

My thesis research is important because it should advance our understanding of predator learning behaviour and prey selection, as well as specifically contribute to our understanding of insect learning behaviour, an area lacking in research efforts. The results from my first experimental chapter (Chapter 2) will provide the first test of the potential ability of crickets to learn a binary food choice using visual cues. Although previous research has demonstrated that crickets are capable of learning (Beugnon 1986; Beugnon \& Campan 1989; Hale \& Bailey 2004; Wessnitzer et al. 2008; Matsumoto \& Mizunami 2000, 2004), no study to my knowledge has yet specifically 
tested whether crickets are capable of using visual cues (namely colour) to successfully learn a binary food choice. The ability of insect predators to successfully respond to a novel food choice is important for our understanding of insect prey selection and its effect on both the predator's and prey's fitness. Regarding predators, the ability of a predator to learn a food preference or a food avoidance can be critical to its survival. By testing whether or not crickets are capable of learning a food preference, we can use this information to further our understanding of crickets' survival in nature. Regarding prey, a cricket's use of visual cues (colour) to learn a food preference or avoidance could directly influence its fitness. Therefore, we can also use this information to explore hypotheses concerning the evolution of prey defence strategies, such as warning coloration (aposematism).

The results from my second data-based chapter (Chapter 3) should also advance our understanding of predator learning behaviour and prey selection. Here, I developed a Bayesian model to explore some of the conditions driving predator learning behaviour, such as the influence of a predator's prior beliefs (Bayesian priors) and the certainty the predator has in its belief on prey selection. One can use the results of such a model (e.g. an optimal sampling strategy) to predict predator learning behaviour in nature and to potentially understand the effect of predator learning behaviour on the evolution of prey coloration (aposematism). Although the effect of a predator's Bayesian priors on the optimal sampling strategy has previously been confirmed (Sherratt, 2011), my model is the first to specifically test the influence of a predator's certainty in its belief on the optimal sampling strategy. Future work could compare my model's predictions to the observed behaviour of predators encountering unfamiliar prey types, and to test whether the certainty of a predator's prior beliefs affects its prey sampling strategy in nature. 


\section{CHAPTER 2}

Investigating learning and prey selection in Jamaican field crickets 


\section{Abstract}

Insect learning behaviour is commonly overlooked, yet insects have been shown to rely considerably on learning for nearly all of life's major events, including spatial navigation, predator avoidance, sexual behaviour, social interactions, and prey selection/foraging. Although learning behaviour in the context of prey selection has been demonstrated for insects, and more specifically for crickets, it is unknown whether crickets are capable of using visual cues to learn a novel binary food choice. Here, I tested the ability of Jamaican field crickets (Gryllus assimilis) to use colour cues as a means of learning to discriminate between a novel rewarding and unrewarding prey. Individual test crickets completed 5 days of learning trials, with each trial comprised of 14 unfamiliar binary food choices. Each binary choice consisted of two prey types, a rewarding prey (1 $\mathrm{mm} \times 1 \mathrm{~mm}$ piece of Gala apple) and an unrewarding prey (a $1 \mathrm{~mm} \times 1 \mathrm{~mm}$ piece of fun foam), each dyed either blue or green. Crickets exhibited a significant increase in the proportion of correct choices (i.e. rewarding prey) made across trials, indicating a learned food preference. Interestingly, the colour of the rewarding/non-rewarding prey significantly affected the proportion of correct choices made. Crickets trained with the rewarding prey coloured green made significantly more correct choices, and overall had a higher attack rate on the rewarding prey throughout. Collectively, my results indicate that crickets (i) are capable of learning a novel binary food choice, and (ii) possess a bias for green coloured prey that influenced learning and prey selection. 


\section{Introduction}

To reduce uncertainties about aspects of their natural environments, individual animals commonly obtain information about their immediate environment through direct personal experience, that is, individual learning (e.g. Dall et al. 2005). For example, they commonly must learn to recognize and avoid predators, where to seek shelter, and how to identify palatable prey. Several strategies have evolved across taxa to maximize learning. One of these strategies is referred to as direct learning, which has been defined as "the modification of behaviour through experience, whereby an animal gains information by interacting directly with its physical environment" (Drea, 2006). Direct learning has been investigated across a wide range of species and has been tested in numerous different contexts. For example, spatial learning and homing behaviour have been investigated in insects and in all major vertebrate taxa (Healy, 1998; Capaldi et al., 2000; Burt De Perera and Guilford, 2008), testing the ability of individuals to learn the location of a significant landmark (e.g. burrow, shelter, hive etc.) based from personal interactions with their environment. Moreover, individual males and females have been shown to learn in the context of mate selection, courtship and sexual behaviour (Shelly and Bailey, 1992; Qvarnstrom et al., 2004; Dukas, 2005 a, b). For example, sexual imprinting has been broadly documented in birds (ten Cate et al., 1993) as well as other taxa (Kendrick et al., 1998), showing that juveniles will acquire sexual preferences through early life experiences, which are later expressed in adulthood (Irwin \& Price, 1999). Equally important, direct learning is key in the context of predator avoidance. It has been reported that many prey species, including but not limited to, certain species of invertebrates, birds, mammals, and fishes, do not show an innate recognition of their potential predators, but rather exhibit learned predator recognition and avoidance (Curio et al., 1978; Mathis et al., 1993; Chivers \& Smith, 1994; Rochette et al., 1997; 
Chivers \& Smith, 1998; Woody \& Mathis 1998; Brown \& Godin, 1999; Griffin et al., 2001; Kelley and Magurran, 2003; Mirza et al., 2006). For instance, many fish species have been shown to acquire recognition of a novel predator through pairing chemical alarm cues with olfactory cues of injured conspecifics, and/or by pairing chemical alarm cues with the corresponding visual or chemical cues of the predator (Chivers \& Smith, 1998; Kelley \& Magurran, 2003; Ferrari et al., 2005, 2007).

Additionally, direct learning plays a critical role in food preference and avoidance. There is sufficient evidence that information gained from personal experience and interactions with prey can lead to learned food aversions and preferences in many predators. Predators across a broad range of taxa have been shown to learn to associate distasteful and/or toxic prey with a defining physical and/or sensory cue exhibited by the prey (Gelperin, 1975; Bernstein et al., 1980; Thorhallsdottir et al., 1987; Mason and Reidinger, 1983; Manteifel \& Karelina, 1996; Mackay, 1977; Taylor et al., 2015). For example, many bird species, including Red-winged Blackbirds (Agelaius phoeniceus), Starlings (Sturnus vulgaris), and Japanese Quails (Coturnix japonica), learn to avoid unpalatable prey after being exposed to a distasteful prey, becoming sick, and consequently associating sickness with the specific visual cues of the prey (Czaplicki et al., 1976; Mason and Reidinger, 1983). This form of associative learning has also been demonstrated in guinea pigs (Braveman, 1975), fishes (MacKay 1974, 1977; Little, 1977; Manteifel \& Karelina, 1996), garter snakes (Burghardt et al. 1973), and chickens (Capretta, 1961), whereby the predator learned to avoid a specific prey type by associating the visual cues of the prey with toxicosis. 
Learned food aversions are widespread across vertebrate taxa and, undoubtedly, the ability of a predator to learn to discriminate between palatable and distasteful and/or noxious prey is important to its survival. However, learning in a foraging context is not limited to vertebrates. Although often perceived as cognitively simpler organisms, invertebrates can also benefit from learning (Dukas, 2008). Evidence of invertebrate learning, specifically in the context of learning a food preference or aversion, has been studied in species in a taxa ranging from molluscs, to nematodes, and to insects. For example, Sahley et al. (1980) reported that the terrestrial mollusc, Limax maximus, learned to avoid food associated with a distasteful odour (quinidine sulfate), showing a markedly reduced preference for the bitter odour compared to the highly attractive odours of potato or carrot. Similarly, Darmaillcq et al. (2004) have also demonstrated aversion learning in a cephalopod, the cuttlefish (Sepia officinalis). In this particular study, the preferred prey of adult cuttlefish was experimentally rendered unpalatable and subsequently presented to the cuttlefish. After exposure to the unpalatable prey, when provided a binary choice between their modified preferred prey and an alternative palatable prey, the cuttlefish learned to avoid their preferred prey and increased attack on the alternative prey. Evidence of learning in invertebrates has also been documented in nematodes. Zhang et al. (2005) demonstrated that after exposure to pathogenic and non-pathogenic bacteria, when completing a binary choice assay, Caenorhabditis elegans showed a learned aversion to odours signalling a pathogenic bacteria, and a learned preference for odours signalling a non-pathogenic bacteria. Finally, evidence of learning has also been reported in insects. Using a similar binary choice design, Lee et al. (2018) demonstrated that fruit flies (Drosophila melanogaster) showed an increased aversion to solutions of high calcium concentration and a preference for sucrose solutions, after exposure to both stimuli. Likewise, Dukas (1999) showed that groups of fruit fly 
larvae (Drosophila melanogaster) learned to prefer odours associated with high-quality food (high protein and carbohydrate content), and avoid odours associated with low-quality food (lacking protein and carbohydrates). Furthermore, Wright et al. (2010) showed that honey bees (Apis mellifera carnica) learned to avoid sucrose-quinine solutions after exposure to both a sucrose-quinine solution and a 100\% sucrose solution. Lastly, Fouks \& Lattorff (2011) demonstrated that, after exposure to parasitic flowers (contaminated by Crithidia bombi) and non-parasitic flowers, bumblebees exhibited an aversion to the parasitic flowers and a preference for the non-parasitic flowers.

Here, I investigated whether orthopterans are capable of learning a food aversion and/or preference using visual discriminatory cues. Although many invertebrates use chemical sensory modalities when interacting with their environment, many species also use visual cues to perform essential tasks, such as identification and discrimination of their surroundings, navigation, and food choice. Indeed, the visual capacity of invertebrates is quite comprehensive. For example, ants have demonstrated the capacity to recognize visual patterns, such as discriminating between vertical and horizontal stripes and between vertical and diagonal stripes (Vowles, 1965).

Moreover, ants have also been shown to distinguish the form of visual cues, using the overall dimension of the cue to discriminate between solid and hollow shapes, as well as to identify the number of elements and the orientation of the cue (Cammaerts, 2008). Likewise, bees have also been shown to discriminate between different visual cues, specifically the ability to discriminate various shapes, including a disc, a square, a diamond, and two different triangles by assessing the position and orientation of the cue's edges (Campan \& Lehrer, 2001; de Ibarra \& Giurfa, 2003). 
The ability of invertebrates to discriminate among different visual cues has important implications for learning. Firstly, visual cues are important for insects learning the location of their nest or a rewarding food source. For example, ants will memorize prominent visual landmarks near their nest to help re-locate their colony after returning from a feeding site (Collett et al., 1992; McLeman et al., 2002). As well, bees will learn the location of a food reward (sugar) by identifying the landmarks surrounding the reward (Cartwright \& Collett, 1982). Secondly, work by Traynier (1984) demonstrated the importance of visual cues for female butterflies learning an oviposition site preference. More specifically, they exposed gravid female Pieris rapae to either a blue or green coloured oviposition site (disc), and 24 hours following the initial contact, the females were then provided a choice between a blue and green disc for oviposition. When tested, the females tended to choose the same coloured site as they had previously been in contact with 24 hours earlier, demonstrating a learned preference for the familiar oviposition site. Lastly, visual cues have also been demonstrated to have an important role in insect foraging. Several studies have shown that butterflies (Weiss, 1997; Swihart \& Swihart, 1970), moths (Kebler, 1996), and locusts (Bernays \& Wrubel, 1985) use colour cues to learn a food preference. In these studies, individuals were trained with a food source (flower) of a given colour that provided a reward, and any/all alternative food source(s) remained unrewarded. In each case, the insect quickly learned to associate the floral colour with the reward, resulting in a strong food and colour preference.

In the present study, I set out to test the ability of Jamaican field crickets, Gryllus assimilis, to learn a dietary preference between a rewarding and unrewarding prey using visual discriminatory cues. One of the earliest accounts of learning in crickets was documented by Campan \& Lacoste (1973). In their study, crickets (Nemobius sylvestris) were trained to 
discriminate the form of two different targets, and subsequently learned a preference for one of these targets by being rewarded with food, water and shelter when approaching it. Research since has continued to explore learning in crickets, with emphasis directed towards testing learning in the context of spatial navigation and homing behaviour (Beugnon 1986; Beugnon \& Campan 1989; Hale \& Bailey 2004; Wessnitzer et al. 2008). In these studies, crickets showed that they were capable of learning the location of their burrow and the correct route to reach it when either the cricket or the nest was displaced from its original position. To my knowledge, only two studies have investigated learning in the context of food selection in crickets (Matsumoto \& Mizunami 2000, 2004). In both studies, the authors investigated the performance of Gryllus bimaculatus in two different discriminatory olfactory learning paradigms. First, the authors tested the ability of crickets to associate one distinct odour with a positive stimulus (water) and associate a second distinct odour with a negative stimulus ( $\mathrm{NaCl}$ solution). It was reported that crickets were capable of making both associations, providing evidence that they have a highly developed olfactory learning capability. Second, the authors tested whether a visual signal, such as the background light condition, had an effect on a cricket's ability to maintain a learned association between a specific odour and food reward. In the latter study, crickets were trained to associate one odour with a positive stimulus and a second odour with a negative stimulus in an illuminated arena. The crickets were then tested in a dark arena to determine whether they would maintain these learned associations when tested under a different background light condition. Again, the authors found that crickets were successful in making both odour and food associations, and maintained both associations when tested in a dark arena.

Considering the above, there is evidence that crickets are capable of learning. However, it has yet to be tested whether crickets are capable of using visual signals to learn a binary food 
choice. Here, I tested the ability of Jamaican field crickets to use colour as a means of learning a binary food choice between a rewarding and unrewarding prey. I hypothesized that crickets should learn a dietary preference by associating the rewarding prey and the unrewarding prey with the corresponding colour of the prey item. The goal of this experiment is to advance our understanding of learning in crickets, specifically in the context of prey selection and visual discrimination. The findings from my experiment can be used to explore further hypotheses regarding the evolution of prey selection and prey coloration, in particular concerning conspicuous coloration and aposematism.

\section{Methods}

\section{Subjects and Housing}

A total of 33 Jamaican field crickets ( 15 females, 18 males) were obtained at adulthood from a laboratory-reared population. The original population was established in September 2007 near the Stengl Lost Pines Biological Station of the University of Texas (Bastrop County, TX, U.S.A; $\left.\sim 3017^{\circ} \mathrm{N}, \sim 9746^{\circ} \mathrm{W}\right)$. Test crickets were housed separately in clear plastic containers $(520 \mathrm{~mL})$ consisting of a screened lid ( $4 \times 4 \mathrm{~cm}$ mesh opening), a shelter (piece of paper towel), and water ad libitum. They were fed powdered Harlan Teklad Rodent diet (no. 8604M; 24.3\% protein, $40.2 \%$ carbohydrate, $4.7 \%$ lipid, $16.4 \%$ fibre, $7.4 \%$ ash) ad libitum preceding Day 1 of trials, after which all food was removed to promote feeding motivation. Individual containers were kept in a greenhouse on a $14 \mathrm{~h}: 10 \mathrm{~h}$ light:dark cycle at approximately $26^{\circ} \mathrm{C}$. All rearing and experiments were conducted at Carleton University in Ottawa, Ontario, Canada. 


\section{Experimental arena}

The experimental arena was constructed of white corrugated plastic, measuring $32 \mathrm{~cm} \mathrm{x} 24 \mathrm{~cm} \mathrm{x}$ $21 \mathrm{~cm}$ (Figure 2.1). The floor of the arena contained 14 binary choice stations, arranged in a 5-45 grid pattern. Each binary choice station measured $2 \times 4 \mathrm{~cm}$ and was constructed out of white card stock paper. Two symbols, a blue circle and a green square, divided each station into halves. Binary choice stations were secured to the arena floor using Velcro. This allowed me to randomize the spatial positioning of the symbols, accounting for any side bias. To facilitate cricket spatial orientation in the arena, each interior-facing wall comprised of a simple visual pattern made from black duct tape. For all behavioural observations, the experimental arena was placed in a $121.92 \mathrm{~cm}$ x $121.92 \mathrm{~cm}$ x $121.92 \mathrm{~cm}$ acoustic chamber lit by two LED lights mounted on opposite facing walls (Figure 2.2). The temperature within the acoustic chamber was maintained constant at approximately $22.3^{\circ} \mathrm{C}$. Observations were recorded using a Logitech HD Pro Webcam (model C920) mounted on the ceiling of the acoustic chamber. 


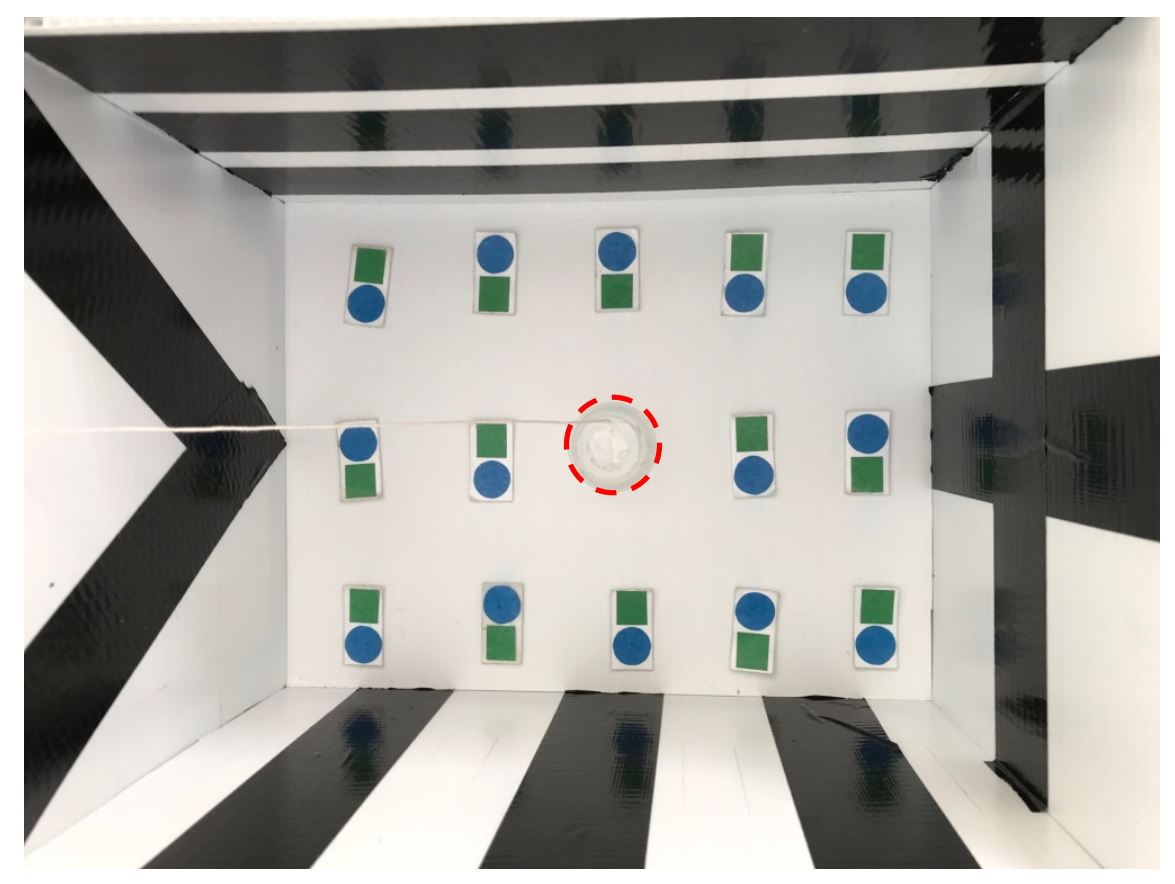

Figure 2.1 Experimental arena $(32 \times 24 \times 21 \mathrm{~cm}, \mathrm{~L} \times \mathrm{W} \times \mathrm{H})$ constructed from white corrugated plastic. Interior walls were distinguishable by patterns of black duct tape; including horizontal stripes, vertical stripes, a V and a cross. The arena included a total of 14 binary choice stations, each consisting of a green square and blue circle. An acclimatization cup in the center of the arena temporarily held a test cricket. A zone of acclimatization was represented by the area under the cup, outlined above by the red circle. 


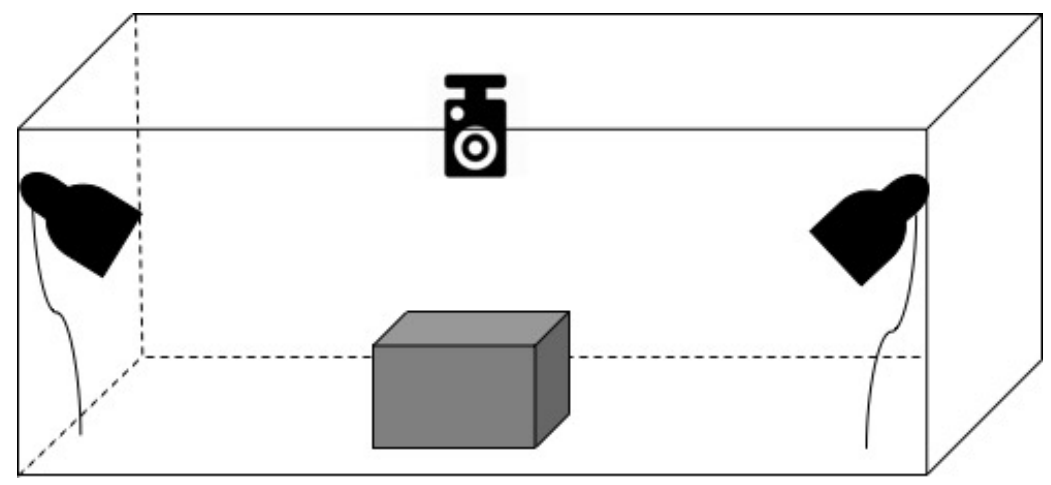

Figure 2.2 Schematic of experimental set up. Acoustic chamber measuring $121.92 \mathrm{~cm} \times 121.92$ cm x $121.92 \mathrm{~cm}$ and lit by two LED lights were mounted on opposite facing peripheral walls. Cricket behaviour was recorded using a Logitech HD Pro Webcam (model C920) mounted overhead. 


\section{Experimental Procedures}

\section{(i) Exploration Period}

Individual test crickets underwent 8 consecutive days of experimental observations. Day 1 and Day 2 of observations constituted a combined 48-h exploration period, consisting of two consecutive 24-h periods. Each exploration period began with a cricket completing a 5-min acclimatization period within the experimental arena. To do this, a cricket was transferred to the center of the arena and placed under a clear plastic cup tethered to a string extending outside of the arena. Following the acclimatization period, the cup was removed and the cricket freed to explore the arena for $24 \mathrm{~h}$. The experimental arena (Figure 2.1) was set up identically to how it would appear for the upcoming training trials, with the exception of the binary choice stations being baited. This was done because the purpose of the exploration period was to acclimatize the crickets to the environment in which they would be trained and tested over the subsequent week. Water and food (powdered Harlan Teklad Rodent diet) were provided ad libitum during the first 24-h exploration period (Day 1), and only water was provided during the second 24-h exploration period (Day 2) to encourage feeding motivation for the following training trials.

\section{(ii) Learning Trial}

Immediately following the exploration periods, individual crickets completed 5 consecutive days of learning trials (Day 3 - Day 7). A learning trial consisted of two parts: a 5-min acclimatization period followed by a 20 -min learning period, initiated once the cricket left the acclimatization zone, defined as the area beneath the acclimatization cup. A learning trial comprised a total of 14 binary choices. Each binary pair included a rewarding food, a $1 \mathrm{~mm}$ x $1 \mathrm{~mm}$ x $1 \mathrm{~mm}$ piece of Gala apple (positive stimulus), and an unrewarding food choice, a $1 \mathrm{~mm} \times 1 \mathrm{~mm} \times 1 \mathrm{~mm}$ piece of fun 
foam (neutral stimulus). Previous work has shown that crickets (Gryllus veletis), show a preference for foods high in carbohydrates, and consume significantly more carbohydrate than protein when given the choice (Harrison et al., 2014). Therefore, given that apple contains a high carbohydrate content, it was chosen as the rewarding food type. To standardize food item size, I used a 1-mm hole punch to cut out all baits. Moreover, to account for differences in olfactory cues, I brushed a piece of Gala apple across each surface of the foam bait before including it in a trial. Crickets were trained to learn the difference between green and blue stimuli, achieved by dyeing each rewarding bait with unscented Club House food colouring, and using blue and green coloured fun foam. These colours were chosen as crickets possess blue (445 nm), green $(515 \mathrm{~nm})$ and ultraviolet $(332 \mathrm{~nm}$ ) photoreceptors, thus allowing discrimination of both blue and green coloration (Zufall et al., 1989). The baits were coloured to match the blue and green symbols on each binary station, and were placed in the center of each station's corresponding symbol. For the purpose of this experiment, the colour blue was always associated with a circle, and the colour green was associated with a square. The station's symbols acted as an alternative visual stimuli for learning, providing crickets the opportunity to discriminate the difference in shape (de Ibarra \& Giurfa, 2003) as well as colour to learn a dietary preference. The colour of the rewarding and unrewarding baits would remain consistent across all trials. Therefore, if a cricket was provided blue coloured rewarding baits in Trial 1, then the rewarding baits would continue to be coloured blue for the remainder of the trials. To control for any previous biases, half of the crickets were trained with the rewarding food dyed blue, and the other half trained with the rewarding food dyed green. Furthermore, the location (left side vs. right side) of the rewarding and unrewarding food at each station was randomized to avoid any side bias. Once a trial was completed, the cricket was removed from the test arena and returned to its individual container 
and housed in a greenhouse until the following day. To remove any/all chemical cues and traces, the arena was sterilized with $95 \%$ ethanol between each trial.

\section{(iii) Test Trial}

On the last day of observations (Day 8), crickets completed a final test trial. The test trial followed the same methodology as a learning trial, with the exception that for the test trial each station consisted of two rewarding choices. Therefore, across all stations, there were 14 blue rewarding food choices and 14 green rewarding food choices. This allowed me to test whether a food preference was learned by visual discrimination while controlling for any olfactory cues.

\section{(iv) Video scoring}

Observations were reviewed using Behavioural Observation Research Interactive Software (BORIS) for Windows on an HP Envy laptop. The following measures were extracted for individual test crickets from the video clips: (1) time taken to leave acclimatization zone, (2) total number of rewarding choices, (3) total number of unrewarding choices, (4) sequence of events and stations visited, (5) time spent at each choice, and (6) the amount of rewarding prey eaten. For the purpose of this study, a food "choice" is defined as a cricket's initial food choice, the first bait attacked at a given binary choice station, and an "event" represents a cricket making a correct (1) or incorrect (0) choice at a given binary station. 


\section{Statistical analyses}

To test whether crickets learned a dietary preference, I fitted a generalized linear mixed-effects model (GLME) to the data. To estimate the parameters of my model, I ran a restricted maximum likelihood estimation. The full model treated trial (as a continuous variable), sequence, the choice number within a trial (as a continuous variable), colour (as a fixed factor), sex (as a fixed factor), the interaction between colour and trial, the interaction between colour and sequence, and individual cricket (as a random factor) as predictors, and the outcome of the event (whether a correct choice (1) or incorrect choice (0) was made) as the binary response variable. Here, I present the most parsimonious model with the lowest Akaike Information Criterion (AIC) among contesting models.

The model treated trial, sequence, colour, sex, and individual cricket as predictors, and the outcome of the event as the binary response variable. If learning occurred, then I expected trial and/or sequence to have significant and positive effects, indicating that crickets made more correct choices as trials progressed, suggesting a learned preference.

To test for an effect of motivation on the probability of crickets making a food choice and hopefully learning a food preference, I fitted a linear model to the data. This model treated the amount of rewarding prey eaten by individual crickets as the response variable, and trial (as a continuous variable), and colour (as a fixed factor) as predictors. If the motivation (hunger level) of crickets affected their probability of learning a food preference, then I expected trial to have a significant effect, indicating that the amount of rewarding prey eaten (apple) depended on the trial number and thus the satiation level of the cricket. 
A third analysis was conducted to assess learning in the Test trial. Here, I fitted a second generalized linear mixed-effects model. However, for this model, I only included data collected from the last training trial (Trial 5), which consisted of rewarding and unrewarding baits, and the final Test trial, which consisted of entirely rewarding baits. This model treated the same variables as predictors, trial (as a fixed factor), sequence (as a continuous variable), colour (as a fixed factor), sex (as a fixed factor), and individual (as a random factor), and maintained the outcome of the event $(0,1)$ as the binary response variable. The purpose of fitting this model is to assess learning in the Test trial, as well to compare learning in the training trials (Trial 5) to the Test trial. For all models, I present the most parsimonious model with the lowest Akaike Information Criterion (AIC) among contesting models. All statistical analyses were conducted in R version 3.4.3 (R Core Development Team 2017) using the lme4 package.

Lastly, to further assess learning across all five training trials and the Test trial, I ran a replicated goodness-of-fit test (G-test) for Trial 1, Trial 5, and the Test trial. Each G-test compared the ratio of correct to incorrect choices made within a particular trial (observed ratio) to an expected ratio of 50:50. The observed ratio was calculated using all choices made within Trial 1 and Trial 5, however, to estimate the observed ratio in the Test trial, only the first choice made was considered. The level of statistical significance (alpha) for multiple G-tests was adjusted using the sequential Bonferroni correction. If a food preference has been learned, then I expected the observed ratio of correct to incorrect choices made within a trial to significantly differ from a 50:50 ratio (i.e. random choice). This would indicate that crickets learned a particular food preference for the correct (rewarding) prey type, and are not attacking prey at random. Note that a significant preference could also suggest crickets knew the correct choice at 
the outset from an alternative stimulus. However, if a food preference has not been learned (or previously known), then I do not expect the observed ratio to significantly differ from the expected ratio, suggesting that crickets are not showing a preference and are attacking prey at random.

\section{Results}

\section{Learning experiment}

Of the original 33 crickets, 28 (13 females, 15 males) successfully completed all 5 days of training. The remaining 5 crickets unfortunately died before completing all trials. Trial number had a significant positive effect on a cricket's food preference (GLMM: $\left.\chi^{2}{ }_{1}=2.16, \mathrm{p}=0.038\right)$, indicating that crickets made more correct choices (rewarding prey) as trials progressed (Table 2.1, Figure 2.3a). There was a marginal positive effect of choice sequence on food preference $\left(\mathrm{GLMM}: \chi^{2}{ }_{1}=3.6, \mathrm{p}=0.06\right)$, providing some evidence that, as crickets encountered more choices within a trial, the probability of making the correct choice increased (Table 2.1, Figure 2.4a). Sex did not have any detectable effect $\left(\mathcal{X}^{2}{ }_{1}=0, \mathrm{p}=0.92\right)$ on the probability of crickets making the correct decision. However, I did find an effect of prey colour $\left(X^{2}{ }_{1}=7.1, \mathrm{p}=0.008\right)$, revealing that crickets trained with rewarding prey coloured green made significantly more correct choices compared to those trained with rewarding prey coloured blue (Table 2.1, Figures $2.3 \mathrm{~b}, 2.4 \mathrm{~b})$. In addition, I tested the interaction between trial and colour, as well as the interaction between trial and sequence, in my model to determine whether the colour of the prey also had an effect on the probability of crickets learning a food preference. I found no significant effect of either interaction (both $\mathrm{p}>0.05$ ), indicating that crickets trained with the rewarding prey coloured green did not show a higher probability of learning a food preference (i.e. a higher slope) compared to crickets trained with the rewarding prey coloured blue. 
Table 2.1 Parameter estimates from fitting via generalized linear mixed-effects model testing for the effects of sequence, trial, colour, and sex on the probability of crickets learning a food preference. Shown are the model's parameters, parameter estimates, standard error values, Z values, and $p$-values for each variable. Statistically significant $p$-values $(p \leq 0.05)$ are boldfaced.

\begin{tabular}{lcccc}
\hline & Estimate & Std. Error & $\mathbf{Z}$ value & p-value \\
\hline Sequence & 0.03172 & 0.01688 & 1.879 & 0.06029 \\
Trial & 0.09079 & 0.04368 & 2.078 & $\mathbf{0 . 0 3 7 6 7}$ \\
$\begin{array}{l}\text { Colour } \\
\text { (Blue) }\end{array}$ & -0.43858 & 0.16571 & -2.647 & $\mathbf{0 . 0 0 8 1 3}$ \\
Sex & 0.01730 & 0.16453 & 0.105 & 0.91626 \\
\hline
\end{tabular}




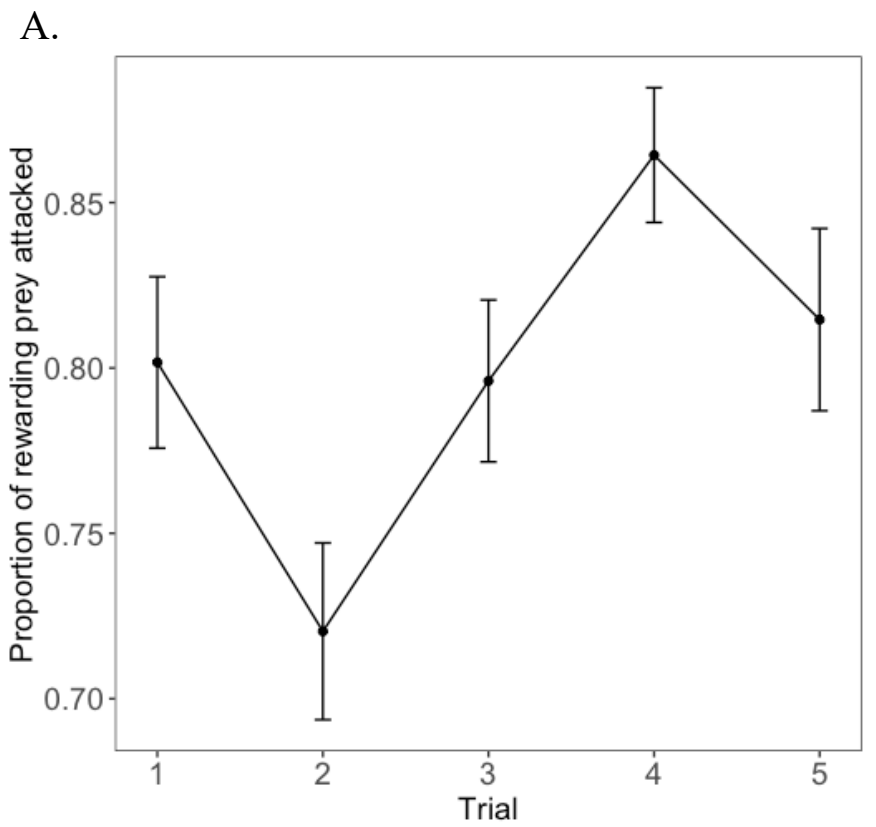

B.

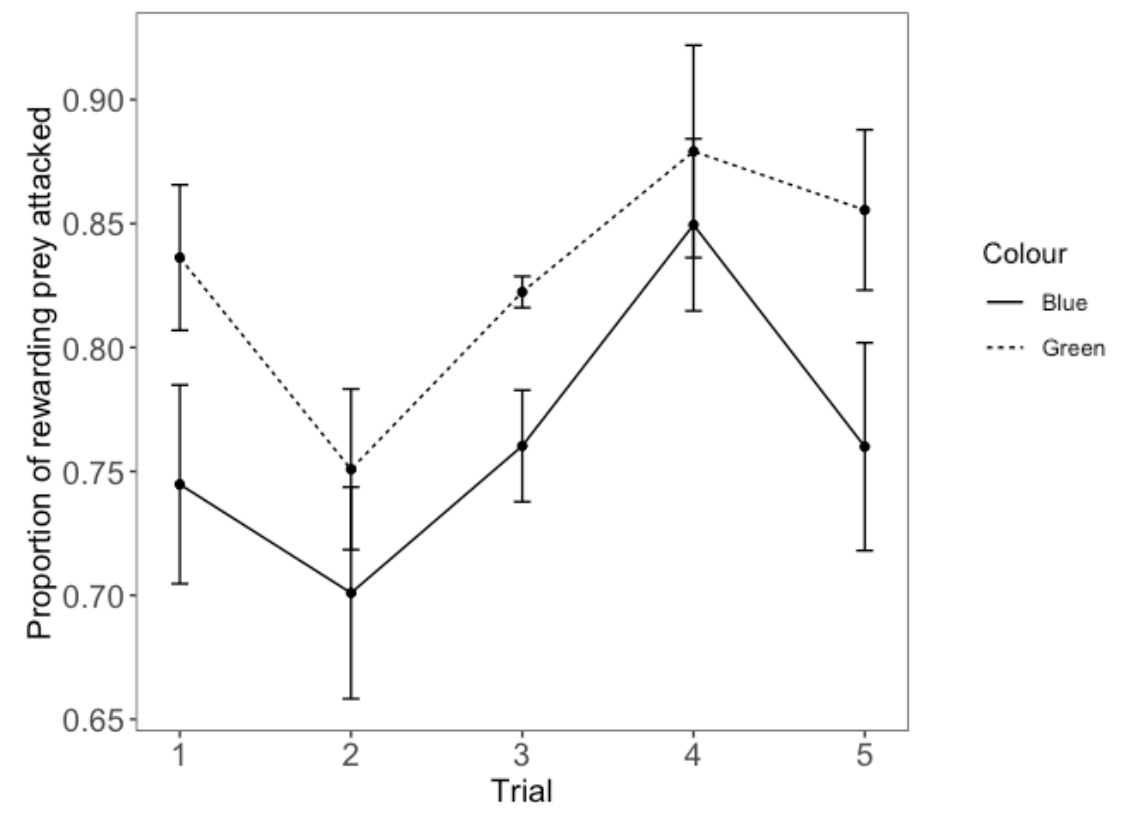

Figure 2.3 Mean proportion of rewarding choices made by adult Jamaican field crickets across 5 consecutive learning trials. A) Mean proportion of rewarding prey attacked for all crickets tested. B) Mean proportion of rewarding prey attacked for crickets trained with the rewarding prey coloured green versus blue. Error bars represent standard error of the mean. 
A.

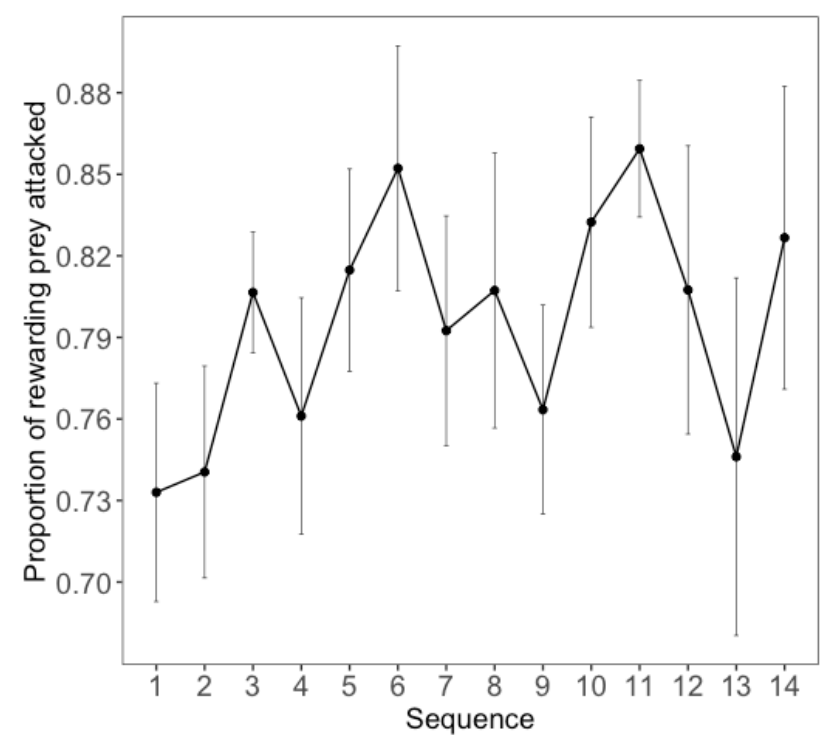

B.

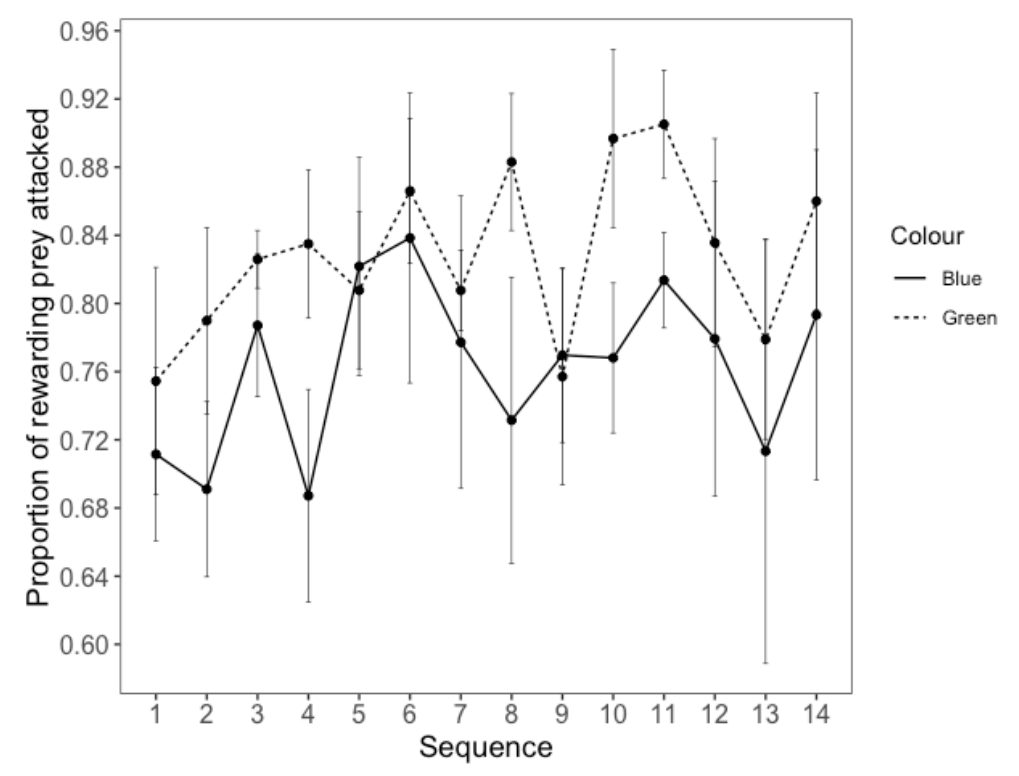

Figure 2.4 Mean proportion of rewarding choices made by adult Jamaican field crickets at each encountered binary choice, spanning from the first to the last binary choice encountered. A) Mean proportion of all 28 crickets across all 5 learning trials. B) Mean proportion of rewarding prey attacked for crickets trained with rewarding prey coloured green versus crickets trained with rewarding prey coloured blue across all 5 learning trials. Error bars represent standard error of the mean. 


\section{Motivation}

To test for an effect of motivation on the probability of cricket's making a food choice and potentially learning a food preference, I fitted a linear model to the data. I found no significant effect of trial on the amount of prey eaten $\left(F_{1,146}=2.12, p=0.146\right)$, suggesting that motivation did not have an effect on my results.

\section{Assessing learning in the Test trial}

To assess the Test data, data extracted from the Test trial was combined with the data of Trial 5 for analysis. There was a significant difference in the proportion of correct choices made between these two trials (GLMM: $\left.X^{2}{ }_{1}=25.29, \mathrm{p}<0.001\right)$, with crickets making significantly fewer formerly "correct" choices in the Test trial (all prey rewarding) compared to Trial 5 (Table 2.2, Figure 2.5). There was a positive effect of choice sequence (GLMM: $\left.\chi^{2}{ }_{1}=2.59, \mathrm{p}=0.02\right)$, providing evidence that more correct choices were made as crickets encountered more binary choices within a trial (Table 2.2, Figure 2.5). Again, sex did not affect (GLMM: $X^{2}{ }_{1}=0.28, p=$ 0.92) the probability of crickets learning a food preference (Table 2.2). However, I did find a similar effect of colour (GLMM: $\left.\chi^{2}{ }_{1}=2.17, \mathrm{p}=0.03\right)$, again demonstrating that crickets trained with rewarding prey coloured green had a greater probability of choosing green prey compared to those trained with rewarding prey coloured blue (Table 2.2, Figure 2.5). 
Table 2.2 ANOVA results from a generalized linear mixed-effects model testing for the effects of sequence, trial, colour, and sex on the probability of crickets learning a food preference in Trial 5 and the Test trial. Shown are the model's parameters, parameter estimates, standard error values, $\mathrm{Z}$ values, and $\mathrm{p}$-values for each variable. Statistically significant $\mathrm{p}$-values $(\mathrm{p} \leq 0.05)$ are boldfaced.

\begin{tabular}{lcccc}
\hline & Estimate & Std. Error & $\mathbf{Z}$ value & p-value \\
\hline Sequence & 0.057 & 0.025 & 2.258 & $\mathbf{0 . 0 2 4}$ \\
Trial & -1.287 & 0.186 & -6.903 & $<\mathbf{0 . 0 0 1}$ \\
$\begin{array}{l}\text { Colour } \\
\text { (Blue) }\end{array}$ & -0.407 & 0.189 & -2.152 & $\mathbf{0 . 0 3 1}$ \\
Sex & 0.141 & 0.188 & 0.748 & 0.455 \\
\hline
\end{tabular}




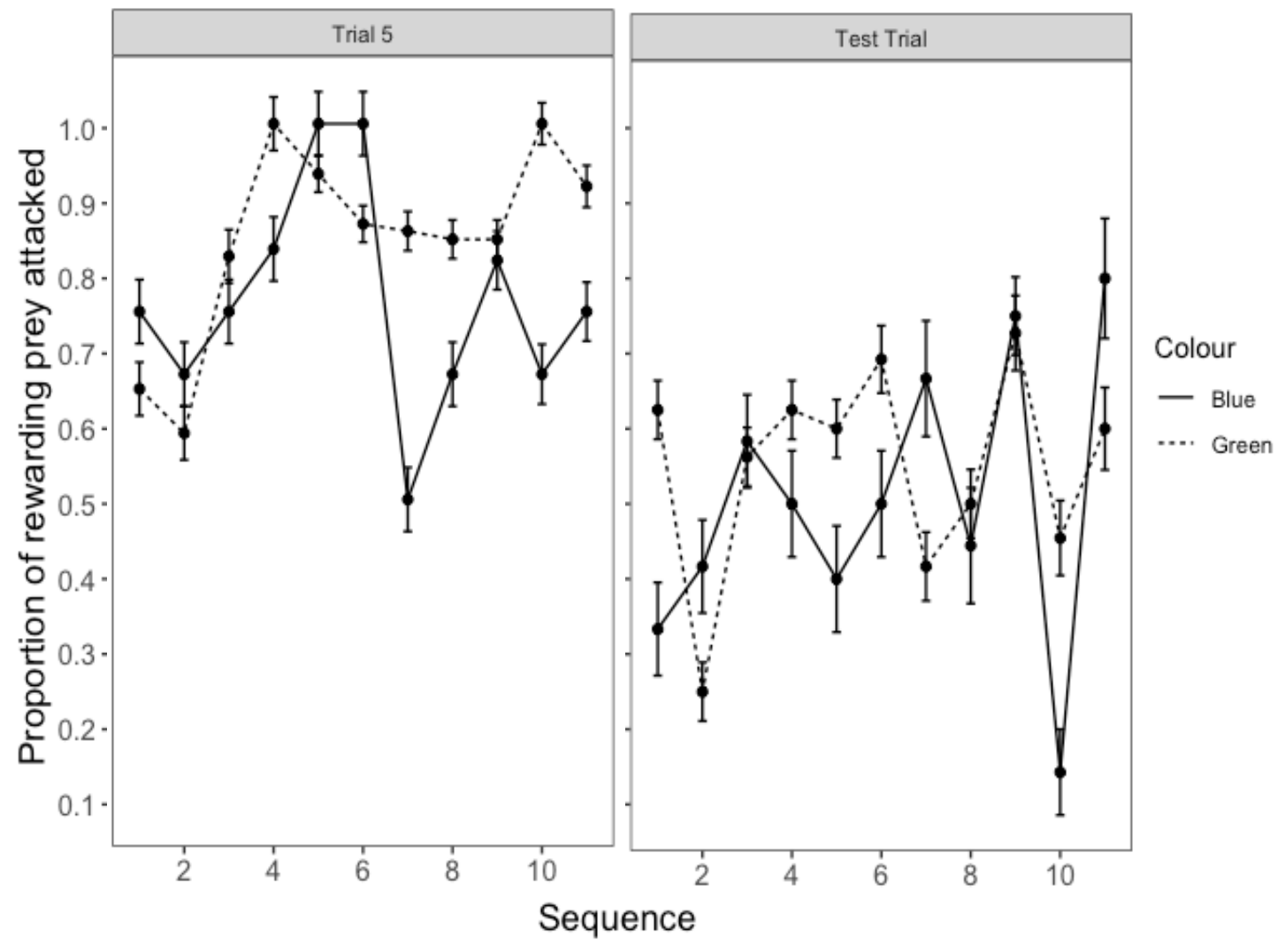

Figure 2.5 Proportion of "rewarding" choices made by all crickets at each binary choice station encountered. Comparison made between Trial 5 and the Test trial. Error bars represent standard error of the mean. 


\section{Comparing learning across all trials}

The observed ratio of the total number of correct and incorrect choices made across all crickets in Trial 1 significantly differed from the expected 1:1 ratio $(\mathrm{G}=137.84, \mathrm{df}=1, \mathrm{p}<0.001)$ (Table 2.3, Figure 2.6). Across all crickets, the mean proportion of "correct" choices made in Trial 1 was $79 \%$. Likewise, the observed ratio of the total number of correct and incorrect choices made across all crickets in Trial 5 also significantly differed from the expected ratio $(\mathrm{G}=134.74, \mathrm{df}=$ 1, $\mathrm{p}<0.001$ ) (Table 2.3, Figure 2.6). In Trial 5, across all crickets, the mean proportion of correct choices made was $81 \%$. Contrary to Trial 1 and Trial 5, the observed ratio of the total number of correct and incorrect choices made at the first binary choice in the Test trial did not significantly differ from the expected ratio of $1: 1(\mathrm{G}=0, \mathrm{df}=1, \mathrm{p}=1)$. As might be expected when all choices are rewarding, the mean proportion of "correct" choices (all stimuli were palatable) made within the Test trial was 50\%. Overall, I can reject the null hypothesis that all of the data from the different trials fit the expected ratio $\left(\mathrm{G}_{\mathrm{total}}=272.58, \mathrm{df}=3, \mathrm{p}<0.001\right)$. As well, I can conclude that overall significantly fewer than $50 \%$ of crickets chose the incorrect prey $\left(\mathrm{G}_{\text {pooled }}=260.22, \mathrm{df}=1, \mathrm{p}<0.001\right)$. 
Table 2.3 Replicated goodness-of-fit tests for Trial 1, Trial 5, and the Test Trial. Shown is the individual G statistic, the total G statistic, the pooled G statistic, degrees of freedom, the observed number of correct and incorrect choices made, and p-value for each test. p-values were adjusted for multiple testing using the Bonferroni method $\left(\alpha_{\text {adjusted }}=0.05 / 9\right)=0.0055$.

Statistically significant $\mathrm{p}$-values are shown in bold $\left(\mathrm{p} \leq \alpha_{\text {adjusted }}\right)$ are boldfaced.

\begin{tabular}{|c|c|c|c|c|c|c|}
\hline & Correct & Incorrect & & $\mathbf{G}$ & df & p-value \\
\hline $\begin{array}{l}\text { Trial } 1 \\
\text { (Sequence:1- } \\
14 \text { ) }\end{array}$ & 287 & 72 & & 137.84 & 1 & $<0.001$ \\
\hline Trained Green & 180 & 36 & & 104.80 & 1 & $<0.001$ \\
\hline Trained Blue & 107 & 36 & & 36.86 & 1 & $<0.001$ \\
\hline $\begin{array}{l}\text { Trial } 5 \\
\text { (Sequence: 1- } \\
14)\end{array}$ & 266 & 63 & & 134.74 & 1 & $<0.001$ \\
\hline Trained Green & 158 & 29 & & 97.89 & 1 & $<0.001$ \\
\hline Trained Blue & 108 & 34 & & 40.53 & 1 & $<0.001$ \\
\hline $\begin{array}{l}\text { Test Trial } \\
\text { (Sequence: } 1 \text { ) }\end{array}$ & 14 & 14 & & 0 & 1 & 1 \\
\hline Trained Green & 10 & 6 & & 1.01 & 1 & 0.31 \\
\hline Trained Blue & 4 & 8 & & 1.36 & 1 & 0.24 \\
\hline & & & Total G & 272.58 & 3 & $<0.001$ \\
\hline $\begin{array}{l}\text { Pooled (across } \\
\text { trials) }\end{array}$ & 567 & 149 & Pooled G & 260.22 & 1 & $<0.001$ \\
\hline
\end{tabular}



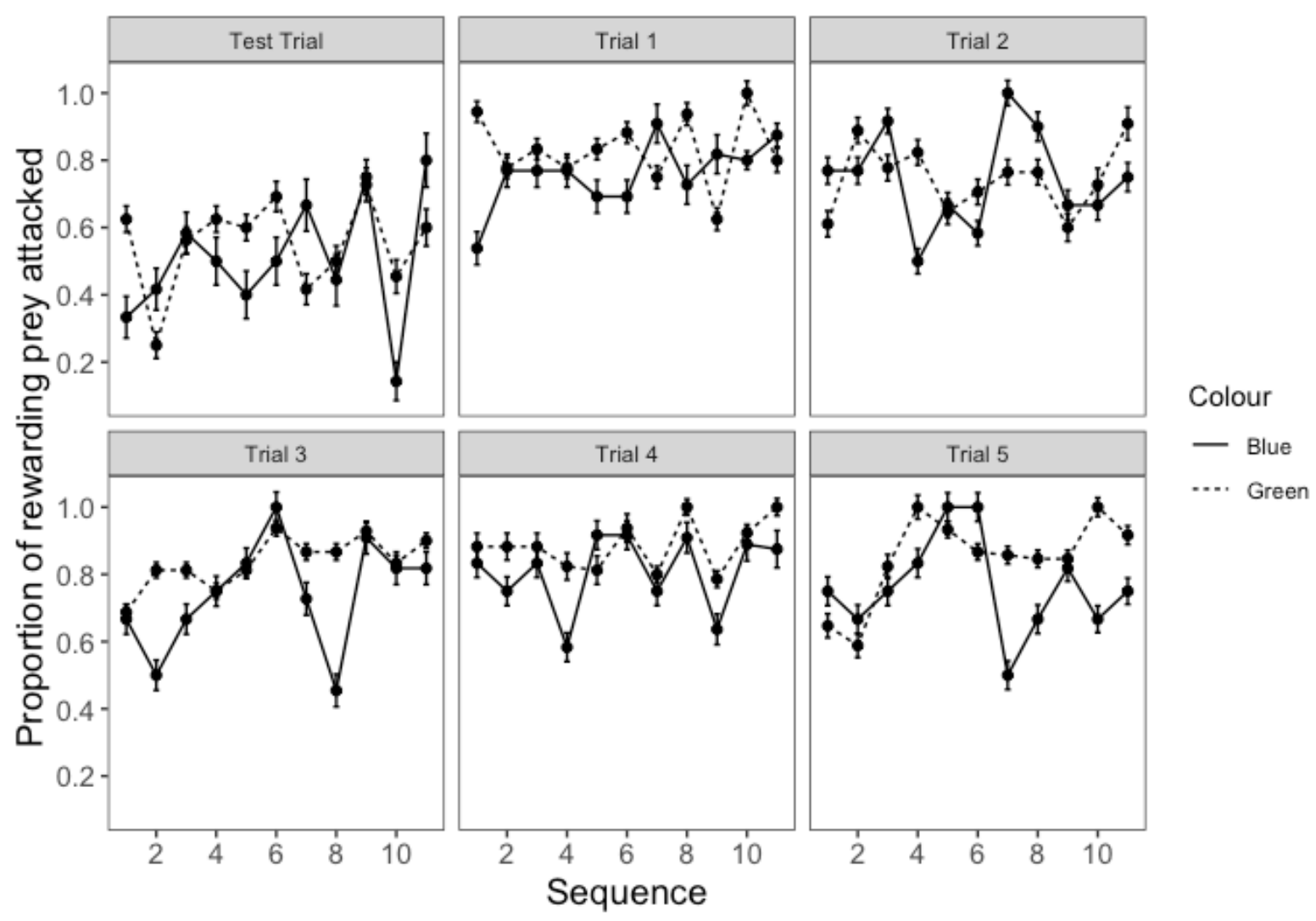

Figure 2.6 Proportion of rewarding choices made by all crickets at each binary choice station encountered. Proportions calculated for each station where a minimum of $50 \%$ of crickets encountered it. Comparison made between Trial 1, Trial 2, Trial 3, Trial 4, Trial 5 and Trial 6 representing the Test trial. Error bars represent standard error of the mean. 


\section{Discussion}

Here, I tested whether Jamaican field crickets are capable of learning a food preference when provided with a series of binary choices consisting of a rewarding and unrewarding novel food item. Overall, my results suggest that, under the conditions of this study, adult Jamaican field crickets are capable of learning a binary food choice. Evidence of learning was first confirmed across trials. As trials progressed from Trial 1 to Trial 5, crickets made significantly more correct choices (Table 2.1), suggesting that they learned a food preference. Figure 2.3 illustrates this trend, showing an overall increase in the proportion of rewarding prey attacked across Trials 1-5. It is important to note that crickets exhibited an initial high attack rate on the rewarding (correct) prey type in Trial 1, overall (Figure 2.3) as well as for the first few choices made within the trial (Figure 2.4). Their initial attack rate (about $75 \%$ ) on the rewarding prey type was much greater than the expected attack rate of $50 \%$ (i.e. no choice) at the outset of the learning trials. This suggests that the crickets possessed a prior bias for the rewarding prey type from the outset, and with increasing experience (across trials) learned to attack the rewarding prey type at a slightly higher rate. Several different factors could be responsible for crickets 'knowing' the rewarding prey type from the start. For example, this initial bias could be the result of an olfactory cue, where despite our controls a difference in olfactory cues between the two prey types still persisted, whether due to an insufficient control (the olfactory cue of the apple is stronger) or the result of the food dye altering the olfactory cues of each prey type. In addition, it could have resulted from visual cues of the rewarding prey at the outset, such as a difference in contrast or texture that allowed crickets to discriminate and identify the rewarding prey from the unrewarding prey. Without further testing, I am unable to reject the possibility that crickets used an alternative cue, or a combination of cues other than colour to learn a food preference. 
Oddly, learning within trials was not confirmed. Thus, our findings did not show a significant effect of sequence, although there was a positive trend (Table 2.1). This result is quite unexpected. We did not expect trial, but not sequence, to have a significant effect. At first glance, a possible explanation for this result could be an effect of the cricket's motivation to learn and to make a food choice within a trial. It is possible that crickets were more motivated (hungrier) at the start of the trial to seek a reward (food) and, as the trials progressed, they became satiated and thus less motivated to seek a food reward. However, this proposition is not supported by the results in Figure 2.4, showing a positive trend of sequence on the proportion of rewarding prey attacked within a trial. As an alternative explanation, it could be that this result is a type II error, and that I failed to reject a null hypothesis that was actually false (false positive).

Interestingly, prey colour had a significant effect on the proportion of correct choices made. My results show that crickets trained with the rewarding prey coloured green made significantly more correct choices compared to crickets trained with the rewarding prey coloured blue, showing overall higher proportions of correct choices made across and within trials (Table 2.1). There are several plausible explanations for this result. First, it could be that crickets possess an innate colour preference for the colour green. Interestingly, not all colour preferences observed in insects are learned. Many studies have revealed that some species possess inborn colour preferences. This idea was first proposed by Charles Darwin (1877), and suggests that instinct and inborn biases will direct an insect to select a particular food source prior to any experience. Evidence of this phenomenon has since been documented in Lepidopteran, Hymenopteran, and Orthopteran taxa. For example, work with Papillonidae (Ilse \& Vaidya, 1956; Weiss, 1997), Pieridae (Traynier, 1984; Scherer \& Kolb, 1987), Nymphalidae (Swihart \& 
Swihart, 1970), and Sphingidae (Kebler, 1996; Goyret et al., 2008) has demonstrated that Lepidopteran species show an innate preference for the colour blue. Likewise, Giurfa et al. (1995) demonstrated a similar preference in honeybees, determining bees to naturally prefer blue and green among a range of 12 colour stimuli. Additionally, evidence of innate colour preferences has also been documented in Orthoptera. Work conducted in naïve grasshoppers, Melanoplus sanguinipes (Bernays \& Wrubel, 1985; Holliday \& Holliday, 1995) and the desert locust, Schistocerca gregaria (Behmer, 2005) demonstrated that both species have an innate preference for the colour yellow. In these studies, grasshoppers and locusts were provided a binary choice between a green and yellow stimulus and, without any prior experience, both species showed an inborn preference for the yellow stimulus.

If crickets possess an innate colour preference for the colour green, I would expect that they would show a significant preference for the green coloured prey on their first encounter with the novel food choice. Therefore, I would expect to see an initial attack rate greater than $50 \%$ for green coloured prey, and consequently an initial attack rate less than $50 \%$ for blue coloured prey in Trial 1 . To test this assumption, I conducted a G-test using only the first choice made by each cricket in Trial 1 to test whether the number of initial attacks on green versus blue coloured prey differed from a 50:50 expected ratio. Seventy four percent of crickets attacked the green coloured prey as their first choice in Trial 1, which is significantly greater that that expected by chance (i.e. $50 \%)(\mathrm{G}=7.572, \mathrm{df}=1, \mathrm{p}=0.006)$. This result provides evidence that Jamaican field crickets showed a significant preference for green coloured prey as their first choice in Trial 1, which can be explained if crickets possess an innate preference for the colour green. 
Second, as an alternative explanation, the reluctance of crickets to associate blue stimuli with palatability could have been influenced by certain innate predispositions associated with warning coloration. Warning coloration (aposematism) refers to bright and conspicuous colours or markings that advertise the unprofitability and/or toxicity of a prey species to predators, consequently deterring predators from attacking (Wallace 1889; Poulton, 1890). Aposematic coloration extends over a wide range of colours and patterns (Schuler \& Roper, 1992; Lindstrom et al., 1999; Lindstrom, 2001; Aronsson \& Gamberale-Stille, 2008; Pegram et al., 2013), all of which strongly contrast the colour of the species background (Joron, 2009). If we consider the terrestrial environment of a cricket, hues of red, orange, yellow and blue would be strongly contrasting against the various hues of green and brown common in its natural environment (Prudic et al., 2006), whereas hues of green would be cryptic, showing very little contrast with background. So, it is reasonable to propose that my test crickets might possess an innate avoidance of aposematic colours, assessing the strongly contrasting blue prey to be aposematic and advertising toxicity or unprofitability. If true, then avoidance of aposematic blue hues could explain why crickets trained to associate blue stimuli (a conspicuous colour) with palatable food made significantly fewer correct choices compared to crickets trained to associate green stimuli (a cryptic colour) with palatable food.

The aforementioned result is also corroborated by the results of Gittleman \& Harvey (1980) and Gittleman et al. (1980). First, Gittleman \& Harvey (1980) demonstrated that male chicks learn to avoid conspicuous distasteful prey more readily than cryptic distasteful prey. In this study, male chicks were fed blue and green coloured crumbs for approximately 3 days. Each chick was subsequently tested in an arena containing 1,000 distasteful crumbs dyed either blue or 
green. Each chick was assigned to one of four treatments; (1) having blue crumbs on a blue background, (2) having blue crumbs on a green background, (3) having green crumbs on a blue background, or (4) having green crumbs on a green background. By the end of the experiment, more blue baits than green were taken on the blue background, as well as more green baits than blue were taken on the green background, suggesting that the chicks more readily learned to associate conspicuous prey compared to cryptic prey with unpalatability. Gittleman et al. (1980) subsequently reinforced this finding, providing additional evidence that vertebrate predators learn to avoid conspicuous distasteful prey more readily than cryptic distasteful prey. In their study, the chicks were offered a choice between blue or green coloured crumbs. The chicks were tested in an arena with 500 blue coloured crumbs and 500 green coloured crumbs in which one colour was made distasteful. Again the chicks were assigned to one of four treatments; (1) having distasteful blue crumbs on a blue background, (2) having distasteful green crumbs on a blue background, (3) having distasteful blue crumbs on a green background, or (4) having distasteful green crumbs on a green background. The test chicks more readily learned to avoid the distasteful crumbs that contrasted the colour of the background compared to the distasteful crumbs that matched, or were cryptic with, the colour of the background. These results may also apply to invertebrate predators, such that these predators show the same innate tendency and more readily learn to associate cryptically coloured prey (green) with palatability compared to conspicuous coloured prey (blue).

Third, the effect of colour on the proportion of correct choices made by crickets could also be due to differences in the cricket's visual sensory system. It is possible that the cricket's visual sensory system allows for better discrimination of green coloured prey compared to blue 
coloured prey, which would explain why crickets trained with the rewarding prey coloured green made significantly more correct choices compared to crickets trained with the rewarding prey coloured blue. The visual system of the cricket is not fully understood. However, Labhart et al. (1984) and Zufall et al. (1988) have demonstrated that crickets (in particular Gryllus campestris and Gryllus bimaculatus) possess three photoreceptors, including blue receptors (445 nm) located specifically in the dorsal rim area (DRA) of the eye, and green (515 nm) and UV (332 $\mathrm{nm}$ ) receptors located in the main dorsal area (DA) of the eye. With these photoreceptors, crickets should be capable of detecting and discriminating blue and green hues. However, it is unknown whether the ommatidia in the DA and DRA differ in their optical properties. Therefore, we cannot rule out the possibility that crickets exhibited a preference for green coloured prey because of differences in their visual acuity and discrimination.

Fourth, it is possible that the effect of colour on the proportion of correct choices made by crickets is due to differences between the two colours of food dye. I can think of two nonmutually exclusive potential differences. One the one hand, blue and green food dyes may have disguised the cues that the crickets used to recognize the rewarding prey type, but to different degrees. For example, it's possible that the blue dye disguised the cues of the rewarding prey (whether visual or olfactory) more heavily compared to the green dye and, as a result, crickets trained with the rewarding prey coloured blue made fewer correct choices. On the other hand, the blue and green food dyes may possess slightly different constituent ingredients and, as a result, the taste and/or olfactory cues from the blue and green dye differ from one another. For example, if the dyes differed in taste, the crickets could have preferred the taste of the green dye compared to the blue dye. As well, if the green dye emitted a more attractive olfactory cue compared to the 
blue dye, it would explain why crickets trained with the rewarding prey coloured green made more correct choices compared to crickets trained with the rewarding prey coloured blue. Assuming there is a difference in olfactory cues between the two food dyes, we can assess whether crickets preferred the olfactory cues emitted by the green dye more than those emitted by the blue dye using the results of the Test trial (Table 2.3). Here, we see that in the Test trial, when all prey were rewarding (half dyed blue and half dyed green), crickets did not show a significant preference for either colour and instead attacked the prey at random. This suggests that if there was a difference in olfactory cues between the two colours of dye, it was not responsible for the observed effect of colour on the probability of crickets learning a food preference.

Finally, we must also consider that the effect of colour on the proportion of correct choices made by crickets goes beyond an initial preference (intercept effect), and instead, the colour of the prey affected the probability of crickets learning a food preference (slope effect). In this case, we would expect the way trial or sequence affects the probability of crickets attacking the correct choice to differ between the two prey colour groups. However, I found no significant effect of the interaction between colour and trial or colour and sequence (both $\mathrm{p}>0.05$ ) on the probability of crickets learning a food preference. This result suggests that the difference in the probability of crickets attacking the correct choice (i.e. between crickets trained with the rewarding prey coloured green versus blue) is the same irrespective of trial number or sequence. Therefore, I can conclude that the colour of the prey did not affect the probability of crickets learning a food preference, and thus crickets trained with the rewarding prey coloured green did 
not have a higher probability of learning a food preference compared to crickets trained with the rewarding prey coloured blue.

While my data confirm a learned food preference in crickets and a significant effect of colour across the training trials (Trials 1-5), I was also interested in assessing learning in the Test trial and comparing learning in the Test trial to the learning observed in the training trials, to determine whether crickets showed and/or maintained a food preference when all prey were rewarding (apple). This was first achieved by restricting my analysis, and analyzing only the data taken from Trial 5 and the Test trial (Table 2.2). This analysis revealed that crickets made significantly fewer correct choices in the Test trial compared to Trial 5, and showed an attack rate that approximated a 50:50 ratio of "rewarding" to "unrewarding" choices in the Test trial, suggesting that crickets did not maintain or show a food preference when all prey were rewarding (Test trial). In addition, I followed up my initial analysis with three separate G-tests for Trial 1, Trial 5, and the Test trial to further compare learning observed across the training trials and the Test trial (Table 2.3). The test comparing Trial 1 and Trial 5 revealed that crickets had a significant preference for the rewarding prey type, thus clearly demonstrating a food preference (Table 2.3). In contrast, the test results for the first choice made in the Test trial showed that crickets did not demonstrate a food preference, and rather attacked the "rewarding" and "unrewarding" prey randomly (Table 2.3, Figure 2.5). Therefore, I can conclude that crickets did not show a food preference in the Test trial, and thus did not maintain their previously learned preference from Trials 1-5. 
Collectively, these findings support my earlier conclusion that Jamaican field crickets could discriminate the rewarding prey (apple) and unrewarding prey (foam) from the outset, either by visual cues, olfactory cues, or a combination of the two. This assumption was first suggested after observing an initial higher-than-expected attack probability on the rewarding prey type in Trial 1, and increasing attack probability in each subsequent training trial (Figure 2.3). Under this assumption, we would expect crickets to show a significant preference for the rewarding prey type in each of the training trials, because using some alternative cue they could identify the rewarding prey type. Furthermore, when crickets were presented with $100 \%$ rewarding prey in the Test trial, we would then expect that they would no longer show a food preference and instead attack prey at random, because using some alternative cue they could identify that each prey was rewarding. This is precisely what we see (Tables 2.2 and 2.3, Figures 2.5 and 2.6). Overall, my results suggest that crickets are capable of successfully responding to a binary food choice and of learning a food preference. The work described here should promote future work investigating learning behaviour in crickets and other insect systems, largely investigating and assessing the use of different sensory cues in learning. 


\section{CHAPTER 3}

An optimal prey-sampling strategy for a predator with Bayesian learning 


\section{Abstract}

Predators that risk attacking unfamiliar prey have the opportunity to benefit from acquiring valuable information about available prey and a potentially profitable meal. However, if the prey has a probability of being unprofitable (harmful or simply unpalatable), then predators are likely to pay a sampling cost. The conditions under which a predator decides to attack, and then to stop attacking, an unfamiliar prey are clearly subject to selection. Here, using a Bayesian model, I formalized the prey-choice problem as an exploration-exploitation trade-off and solved for the optimal sampling strategy for a predator assuming Bayesian learning. First, I set out to identify how a predator's prior beliefs (Bayesian priors) influence prey selection and learning. My model revealed that a predator's previous beliefs concerning the palatability of a novel prey can affect its decision to attack, and consequently determines the optimal sampling strategy. Second, I used this model to investigate how a predator's certainty in its prior beliefs influences the optimal sampling strategy. The certainty a predator has in its prior beliefs is represented by the variance, the dispersion around the mean expected value of its prior. I found that both the expectation and the variance around a predator's prior can affect its optimal sampling strategy. Third, I compared the model's optimal sampling strategy with the results of my previous learning experiment (Chapter 2) using Jamaican field crickets (Gryllus assimilis). The theoretical and empirical learning strategies did not match because of the high probability of crickets choosing palatable prey items at the outset of the cricket learning experiment. Collectively, my results indicate that (i) a predator's previous beliefs concerning the palatability of an unfamiliar prey affect its decision to attack, (ii) the certainty a predator has in its prior beliefs also affects its decision to attack and, (iii) the results from my previous experiment using Jamaican field crickets did not 
conform to my model's optimal sampling strategy, suggesting that unknown factors may have influenced my experiment. 


\section{Introduction}

When confronted with a potential prey, a predator must decide whether or not to attack it.

Choosing to attack is both a gamble and a guarantee; a gamble because, if the prey is palatable, the predator profits from a meal, but at a potential cost if the prey is toxic/harmful, and a guarantee because, regardless of the palatability of the prey, the predator gains information by attacking. Strictly speaking, choosing not to attack is also a guarantee because the predator will not face any added benefits or costs and will also not gain any valuable information. This problem is further complicated when a predator is confronted simultaneously with a choice of two or more prey types. Now, the predator must not only decide whether or not to attack an encountered prey, but must also decide which of the available prey is most profitable. To solve this problem, typically some degree of exploration by the predator is required, an opportunity for the predator to explore and test out its alternatives before confidently choosing and exploiting a single choice.

In the above scenarios, a predator is faced with an exploration-exploitation trade-off, also referred to as the multi-armed bandit problem (Berry \& Fristedt, 1985, see Chapter 1). The multiarmed bandit problem describes an hypothetical situation in which an individual is faced with a slot machine (or "bandit") with multiple levers, and each lever provides a reward chosen at random from a given distribution (Berry \& Fristedt, 1985). The goal of the decision maker is to maximize the sum of his/her rewards. Initially, the individual may pull multiple levers (i.e. sample/explore) to estimate each lever's payoff. However, at some point, the individual should stop exploring and instead use the information he/she has gathered to exploit the lever estimated to have the greatest reward. Here, just like our predator, the individual must decide whether to 
continue exploring or to stop exploring and instead use the information he/she has gathered to exploit the estimated best choice. Explore too long and the individual will be well informed, but will not have used his/her accumulated information. Alternatively, explore too short a time and the individual could be missing out on a far better option. Bandit models are formulated to identify the payoff-maximizing solution to this trade-off.

It should be emphasized that exploration-exploitation trade-offs are not strictly limited to predators and prey selection. For example, Pratt and Sumpter (2006) provide evidence of exploration-exploitation trade-offs extending to emigration and nest relocation in ant colonies, showing how colonies of Temnothorax curvispinosus would explore a number of alternative nesting sites before choosing to abandon their current nest for the nest estimated to be most profitable. Furthermore, exploration-exploitation trade-offs have also been applied to resource patch choice (Krebs et al., 1978; Thomas et al., 1985; Plowright \& Plowright, 1987). One example of this can be seen in foraging bumblebees, where individual bees would repeatedly visit flower species that differed in food reward before committing to the most rewarding flower (Keaser et al., 2002). Lastly, exploration-exploitation trade-offs have even been extended to unicellular organisms lacking a brain. Reid et al. (2016) showed that unicellular slime mould would continue to explore different environments ( $1 \%$ blank agar or 5\% oat-agar) before eventually exploiting the environment deemed most favourable (i.e. provided the most rewarding food sites). Regardless of the context, the solution to an exploration-exploitation trade-off involves finding the balance between exploration and exploitation that maximizes reward, that is, the optimal sampling strategy. This strategy describes the payoff maximizing combination of time an individual should spend exploring its alternatives versus the time it should spend 
exploiting the best choice, with the overarching goal to maximize the sum of the individual's rewards. Naturally, the solution is subject to change as more information is gathered by the individual.

Predators have been shown to demonstrate biases leading to both the avoidance and/or preference of certain prey phenotypes. For example, many predator species have been observed to avoid species displaying aposematic coloration, that is, bright and/or conspicuous colours or markings that warn and deter predators. Evidence has shown that predators learn to avoid brightly coloured prey more quickly than cryptic prey (Schuler \& Hesse, 1985; Paradise \& Stamp, 1991; Speed, 2000), or that predators will innately avoid conspicuous prey without any prior exposure or experience (Roper, 1990; Mastrota \& Mench, 1995; Lindstrom, 1999). Moreover, predators have also been shown to possess innate preferences. Spontaneous colour preferences have been documented across vertebrate (Salzen \& McKeown, 1971; Schmidt \& Schaefer, 2004; Spence \& Smith, 2008) and invertebrate taxa (Ilse \& Vaidya, 1956; Swihart \& Swihart, 1970; Giurfa et al., 1995; Behmer, 2005), demonstrating that predators with no previous experience will exhibit an innate preference for prey of a certain colour. Here, I am interested in investigating how such previous biases affect a predator's decision to attack, and/or to continue attacking, an unfamiliar prey type, and overall how a predator's previous biases affect the optimal sampling strategy.

A predator with a previous bias concerning the profitability of unfamiliar prey, will also have a level of certainty in its belief, in other words, a degree of confidence that the predator's prior bias is correct. The certainty a predator has in its prior beliefs is represented by the 
variance, the dispersion around the mean expected value. If a predator is confident that its prior belief concerning the palatability of a prey is true, the variance around this expectation will be small, representing the predator's high degree of certainty in its prior knowledge. Conversely, if a predator has less confidence in its prior belief, the variance will be larger, representing the predator's low degree of certainty in its prior knowledge. It is likely that the number of unfamiliar prey sampled by a predator will influence the predator's variance around its mean expectation. In particular, the more unfamiliar prey that a predator samples that confirms its mean expectation, should increase the predator's certainty in its belief (lower the variance), whereas the more unfamiliar prey that a predator samples that refutes its expectation, should decrease the predator's certainty in its belief (increase the variance). In this case, it is important to note that ecological factors such as the predictability of the environment, as well as the natural variation in the relative encounter rates of different prey types, may have an effect on a predator's certainty in its belief. However, such hypotheses have yet to be tested. To date, the certainty a predator has in its prior expectation, more specifically, whether or not the predator's certainty affects a predator's decision to attack, and/or to continue attacking an unfamiliar prey type, has yet to be investigated. Therefore, in this study, I set out to investigate whether the certainty a predator has in its prior beliefs has an effect on the predator's optimal sampling strategy.

My primary goal in developing a dynamic Bayesian prey-choice model here was to ascertain how a predator's prior beliefs (Bayesian priors) might influence prey selection and learning. I was interested in identifying the optimal sampling strategy which maximizes a predator's reward, while accounting for the predator's prior beliefs, the cost incurred by 
sampling an unpalatable prey, the benefit gained by sampling a palatable prey, and the total number of available prey. My secondary aim was to use this model to investigate how a predator's certainty in its expectations influences the optimal sampling strategy. More specifically, I asked how does the variance around a predator's mean expected prior influence its decision to attack and to keep attacking an unfamiliar prey? Lastly, by developing such a model, I was able to gain some insights into the actual prey-choice decision making of live animals by comparing the optimal sampling strategy identified by my model against the observed prey choices made by Jamaican field crickets (Gryllus assimilis) in a previous experiment (Chapter 2).

\section{Methods}

All simulations and analyses were conducted using R 3.4.3 (R Core Development Team 2017).

See Appendix A for the code of my Bayesian prey-choice learning model.

\section{Model: Overall approach}

Here, I adopted a Bayesian approach to identify the optimal learning strategy for a predator faced with a novel binary food choice. In my model, a predator begins with prior knowledge (i.e. "belief") of the likely palatability of each prey type and subsequently updates its knowledge by sampling the prey and gaining information, which in turn shape its posterior beliefs. After exploring the different prey types, eventually a predator will decide to stop exploring and instead use the cumulative information it has gathered to exploit the most profitable prey type. The moment a predator stops exploring and instead begins to exploit a single prey type (or no prey at all) is referred to here as the optimal switching point. Likewise, the total number of prey a predator samples before reaching the optimal switching point is referred to as the critical number of prey consumed. Using dynamic programming and an appropriate learning model, I identified 
an optimal learning strategy, and pinpointed the optimal switching point and the critical number of prey a predator should consume when faced with an unfamiliar binary prey choice.

\section{Description of the model}

\section{Calculating Bayesian priors $\alpha$ and $\beta$}

In adopting a Bayesian modelling approach, a necessary first step is to address the predator's Bayesian priors. In other words, we need to describe the predator's prior beliefs concerning the probability of an unfamiliar prey item being palatable. This is achieved using a hypothesis distribution. One such hypothesis distribution is a beta distribution, recognized in Bayesian inference as the conjugate prior probability distribution of the binomial - thus the prior and posterior will both follow beta distributions, albeit with different parameter values (Bolker, 2008). The beta distribution is a probability distribution ranging from 0 to 1 , defined by two parameters $\alpha$ and $\beta$. These two parameters ( $\alpha$ and $\beta$ ) establish the shape of the prior probability distribution, together summarizing the predator's prior beliefs (Bayesian priors).

We need to calculate $\alpha$ and $\beta$ to estimate the prior probability distribution. For the purpose of my model, $\alpha$ and $\beta$ were calculated based on the mean $(\mu)$ and variance $\left(\sigma^{2}\right)$ of the distribution. In a beta distribution, the mean and variance are defined as follows, $\mu=\frac{\alpha}{\alpha+\beta}$ and $\sigma^{2}=\frac{\alpha \beta}{(\alpha+\beta)^{2}(\alpha+\beta+1)}$. So, to calculate $\alpha$ and $\beta$, we solve for $\alpha$ and $\beta$ in both equations and find $\alpha=\frac{-\mu\left(\sigma^{2}+\mu^{2}-\mu\right)}{\sigma^{2}}$, and $\beta=\frac{\left(\sigma^{2}+\mu^{2}-\mu\right)(\mu-1)}{\sigma^{2}}$. Consequently, this sets an upper limit on the variance, $\sigma^{2}<\mu(1-\mu)$ and, thus for a given population mean, the variance cannot exceed a certain maximum. Next, using these two equations, we can calculate the priors ( $\alpha$ and $\beta$ ) corresponding to a pre-determined mean and variance of the probability distribution. I used this 
approach here as it integrates all available information to describe the prior probability distribution, incorporating both the predator's prior expectation $(\mu)$ and the confidence in its expectation $\left(\sigma^{2}\right)$. By taking this approach, we are aware of both the mean and variance and are able to control both of these variables to best describe the prior probability distribution, overall providing greater biological relevance.

\section{Calculating the posterior}

Continuing with a Bayesian approach, we must consider that the prior probability will be updated to a posterior probability in light of newly acquired experience and information about the available prey. As previously mentioned, in a beta distribution the expectation is given by

$\frac{\alpha}{\alpha+\beta}$. Given that the prior probability followed a beta distribution, the resulting posterior distribution will also follow a beta distribution, and can be defined as $\frac{r+\alpha}{n+\alpha+\beta}$, where $(n)$ represents the total number of prey sampled, and $(r)$ represents the number of prey sampled that were unpalatable. Here, we can see how new evidence is incorporated to update a predator's prior expectation concerning the probability of an unfamiliar prey being palatable.

To further illustrate this concept, let's consider a situation where we set $\alpha=1$ and $\beta=1$, generating a prior probability of 0.5 . In this case, the predator believes the likelihood that an unfamiliar prey is unpalatable is 0.5 . Now, let's assume the predator samples 10 unknown prey $(n)$, and 8 of these prey turn out to be unpalatable $(r)$. This generates a posterior probability of $(8+1) /(10+1+1)=0.75$. At this point, the predator now believes the likelihood that an unfamiliar prey is unpalatable is 0.75 (the variance around this expectation also changes). 
Therefore, we can see that a predator's prior beliefs are updated to a posterior probability after gaining new experience and information.

\section{Finding the critical switching point}

We now know how a predator's beliefs will be updated as more prey are sampled. An important relevant question is at what point should a predator stop exploring the different prey types and instead start exploiting the optimal choice? The answer lies in finding the critical number of prey a predator will sample before complete avoidance of any/all prey believed to be suboptimal to sample. In the current Bayesian model, we will assume that the total number of unfamiliar prey a predator will encounter is known, represented by $N$. Again, we will use $n$ to represent the total number of prey sampled, and $r$ to denote the number of prey sampled that were unpalatable. Together, these variables $(r, n)$ encapsulate all of the ecological information a predator has gained about the available prey, representing a predator's present knowledge state.

For any given knowledge state, let $S(r, n)$ represent the maximum benefit return to the predator from deciding optimally in the remaining $(N-n)$ trials. Moving forward from its knowledge state, a predator has the choice to attack or not attack future encountered prey. This behavioural choice will consequently result in a unique return to the predator. I assigned the subscript "A" to represent a predator attacking the unfamiliar prey, $S_{\mathrm{A}}(r, n)$. A predator's choice to attack can be seen as a risk; on one hand, attacking provides the predator additional knowledge regarding the palatability of the unfamiliar prey, as well as providing an opportunity to gain an immediate reward if the prey is palatable, but, on the other hand, if the unfamiliar prey is unpalatable, then an immediate cost is incurred. To represent a predator not attacking a prey 
(deferring attack), I assigned the subscript " $\mathrm{D}$ ”, $S_{\mathrm{D}}(r, n)$. In any case, when a predator defers attack there is no opportunity of gaining additional knowledge, nor any opportunity for immediate benefit or cost. Therefore, we can set $S_{\mathrm{D}}(r, n)=0$, as there is no return and no reason to sample further and thereby gain information.

The goal is to determine the maximum return, $S_{\max }(r, n)$, from all remaining trials $(N-n)$ and for any given knowledge state $S(r, n)$. Therefore,

$$
S_{\max }(r, n)=\max \left[S_{\mathrm{D}}(r, n), S_{\mathrm{A}}(r, n)\right]
$$

where

$$
S_{\mathrm{D}}(r, n)=0
$$

and

$$
S_{\mathrm{A}}(r, n)=p(S[r+1, n+1]-c)+(1-p)(S[r, n+1]+b)
$$

Here, $p$ represents the expectation that the next prey item sampled is unpalatable, otherwise known as the Bayesian prior mean estimate of $\mathrm{P}, p=(r+\alpha) /(n+\alpha+\beta)$ for a given $r$ and $n$ (Sherratt, 2011; Aubier \& Sherratt, 2015 ). Using dynamic programming, I solved for the optimal sampling strategy (whether the predator attacks or defers attack) by backward iteration (Mangel \& Clark, 1988; Clark \& Mangel, 2000). Here, we know for the absolute maximum trial number $N$ that $S(r, N)=0$, since a predator who defers attack will no longer gain any information. Given $S(r, N)=0$, we can calculate $S(r, n)$ by working backwards from $n=N$ (solving for $S(r, N-1), S(r, N-2)$, etc.). Lastly, using forward iteration, we can predict the 
behaviour of the predator as a function of its current knowledge state, assuming that the predator always adopts the optimal strategy.

\section{Results and Discussion}

Here, I have asked whether a predator's prior knowledge of available prey types in the environment (i.e. Bayesian priors) affects its decision to attack or ignore an encountered unfamiliar prey and whether to keep attacking an unfamiliar prey type. To answer this question, I formalized this problem as an exploration-exploitation trade-off and solved for the optimal sampling strategy for a predator assuming Bayesian learning (Sherratt, 2011; Aubier \& Sherratt, 2015). As expected, my model revealed that a predator's prior beliefs concerning the palatability of a novel prey does affect its decision to attack, and consequently affects the optimal sampling strategy (Figure 3.1). Figure 3.1 shows the optimal sampling strategy for a predator starting with a specific combination of Bayesian priors ( $\alpha$ and $\beta$ ), and with the cost of attacking an unpalatable prey $(c)$ and the benefit of attacking a palatable $(b)$ prey set at 1 , and the total number of available prey fixed at 14 (as was the case in my experiment described in Chapter 2). Each sampling strategy is characterized by an optimal switching point, representing the point at which a predator should stop attacking the unpalatable prey type and completely reject it moving forward. To quantify the optimal switching point, I referred to the critical number of prey a predator consumes before reaching this point of complete avoidance. My model's results show that a predator with the previous belief that the majority of all unfamiliar prey are unpalatable ( $\alpha$ $>\beta$ ) will have a more conserved sampling strategy, and will attack fewer unfamiliar prey before reaching the optimal switching point. In contrast, a predator with the previous belief that the majority of all unfamiliar prey are palatable $(\alpha<\beta)$ will have an extended sampling strategy, and 
will attack more unfamiliar prey before reaching the optimal switching point. For example, assuming that a predator has a negative bias concerning the palatability of unfamiliar prey, and set $\alpha=2, \beta=1$ (Figure 3.1), the optimal switching point is reached after sampling a single unpalatable prey. However, if the predator's priors are reversed such that it now has a positive bias concerning the palatability of unfamiliar prey and if we set $\alpha=1$ and $\beta=2$ (Figure 3.1), then the optimal sampling strategy is extended and the optimal switching point is now reached after sampling 3 unpalatable prey. This trend is exaggerated when the strength of the predator's belief is increased and set $\alpha=5$ and $\beta=1$ (Figure 3.1), or $\alpha=1$ and $\beta=5$ (Figure 3.1). Here, we see that when a predator has a strong negative bias concerning the palatability of unfamiliar prey, the predator immediately rejects the novel prey without risking an attack. However, conversely, when a predator has a strong positive bias concerning the palatability of unfamiliar prey, the optimal sampling strategy is much more persistent, and the optimal switching point is reached after sampling approximately 9 unpalatable prey. To further exaggerate this effect, if we set $\alpha=$ $1, \beta=10$ (Figure 3.1), we can see that with an even stronger positive bias the predator nearly attacks all 14 available unpalatable prey, reaching the optimal switching point after consuming approximately 12 prey.

Based on the Bayesian approach adopted here, the observed results above are exactly those we would expect. A predator adopting Bayesian learning begins with an initial belief concerning the palatability of unfamiliar prey, and as the predator begins to gain information about available prey types its initial beliefs are updated. Under this condition and on the one hand, a predator with the prior belief that unfamiliar prey are unpalatable will attack fewer unpalatable prey, and will completely avoid attacking unfamiliar prey sooner because this 
conforms to their prior expectation. On the other hand, a predator with the prior belief that unfamiliar prey are palatable will attack more unpalatable prey, and will hold off completely avoiding attack because this opposes their prior expectation, and therefore the predator will require more evidence (a greater critical number of prey consumed) to update its initial expectation. 


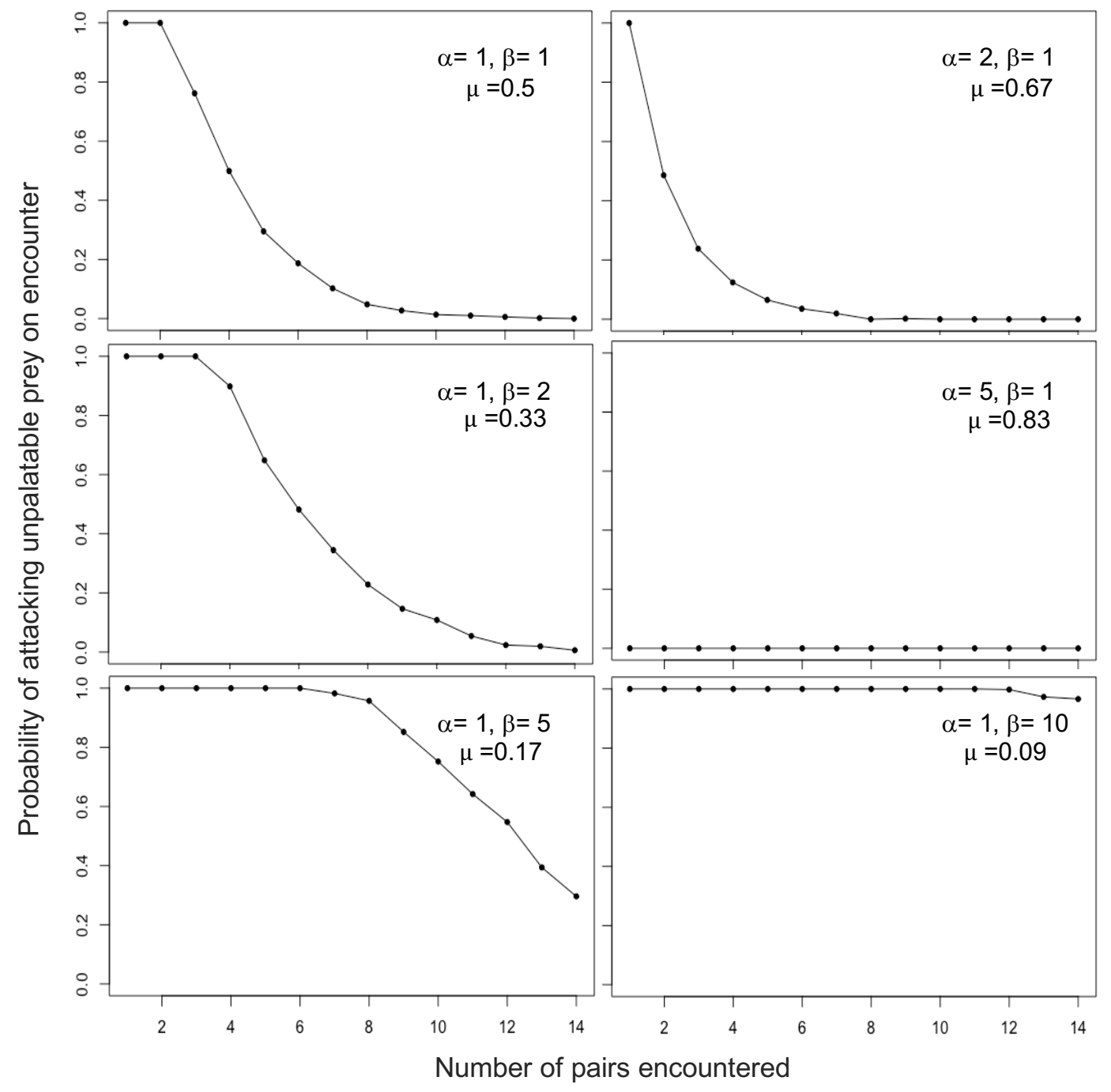

Figure 3.1 The optimal sampling strategy for a predator assuming Bayesian learning, with set Bayesian priors ( $\alpha$ and $\beta$ ) and mean expectation $(\mu)$ concerning the palatability of 14 unfamiliar prey. 
Having observed an effect of a predator's prior beliefs on its decision to attack and/or to keep attacking a novel prey type, my second aim was to investigate whether the certainty a predator has in its beliefs also affects its sampling strategy. The certainty a predator has in its prior beliefs is represented by the variance, the dispersion around the mean expected value. If a predator is confident that its prior belief concerning the palatability of a prey is true, the variance around this expectation will be small, representing the predator's high degree of certainty in its prior knowledge. Conversely, if a predator has less confidence in its prior belief, the variance will be larger, representing the predator's low degree of certainty in its prior knowledge. Figure 3.2 shows the critical number of prey consumed by a predator as the predator's prior expectation and certainty in its prior expectation increase, and where the cost (c) of attacking an unpalatable prey and the benefit $(b)$ of attacking a palatable prey are both set at 1 , and the total number of available prey is fixed at 30 . Note that in this example again we are assuming simple conditions where the predator is faced with a single unknown prey, but now with a total of 30 sampling opportunities. Here, we can see two trends (Figure 3.2). First, as a predator's prior expectation that a prey is unpalatable increases (believing the prey to be more distasteful/toxic), the critical number of prey a predator should consume decreases. This trend is analogous to our results from Figure 3.1, demonstrating that a predator's Bayesian priors can affect its optimal sampling strategy and, in particular, having a negative bias concerning the palatability of a prey decreases the critical number of prey a predator should consume before avoiding attack (the optimal switching point). Second, the variance around a predator's prior expectation also has an effect on its optimal sampling strategy. A predator starting with a positive bias concerning the palatability of the unfamiliar prey will consume more prey given a high certainty (low variance) in its belief, compared to that of a predator with a low certainty (high variance) in its belief. Likewise, a 
predator starting with a negative bias concerning the palatability of the unfamiliar prey will consume fewer prey given a high certainty (low variance) in its belief, compared to that of a predator with a low certainty (high variance) in its belief.

For example, here, the optimal sampling strategy for a predator with a prior belief of 0.3 that unfamiliar prey are unpalatable, and a variance of 0.01 , should be to consume approximately 28 prey before avoiding attack, whereas that same predator, now with a variance of 0.08 , should only consume approximately 6 prey before avoiding attack. In this example, as the predator becomes less confident in its previous bias (i.e. increased variance), the critical number of prey it is expected to consume decreases, resulting in a more conservative sampling strategy despite the predator's mean expectation being quite low. The opposite can be seen for a predator starting with a prior belief of 0.8 that unfamiliar prey are unpalatable (Figure 3.2). In this example, if the predator starts with a variance of 0.01 , it should consume approximately 1 prey before rejecting the prey altogether, whereas if the predator began with a variance of 0.08 , it should sample more and attack about 4 prey before avoiding attack. Intuitively, these trends are again precisely what we would expect to see of a Bayesian learner. One would expect that a predator starting with the prior belief that the majority of prey are unpalatable, and who has little certainty in its belief, will attack more prey because the uncertainty it has in its belief provides reason to believe that its initial belief is wrong and in actuality the majority of prey are profitable. Therefore, the predator will sample more prey as it has more to gain should the majority of prey end up being palatable. The same logic can be applied for a predator starting with the prior belief that the majority of prey are palatable. In this case, one would expect that a predator who has little certainty in its 
belief will attack fewer unpalatable prey, because again its uncertainty provides reason to believe that its initial belief is wrong, and in actuality the majority of prey are unprofitable. 


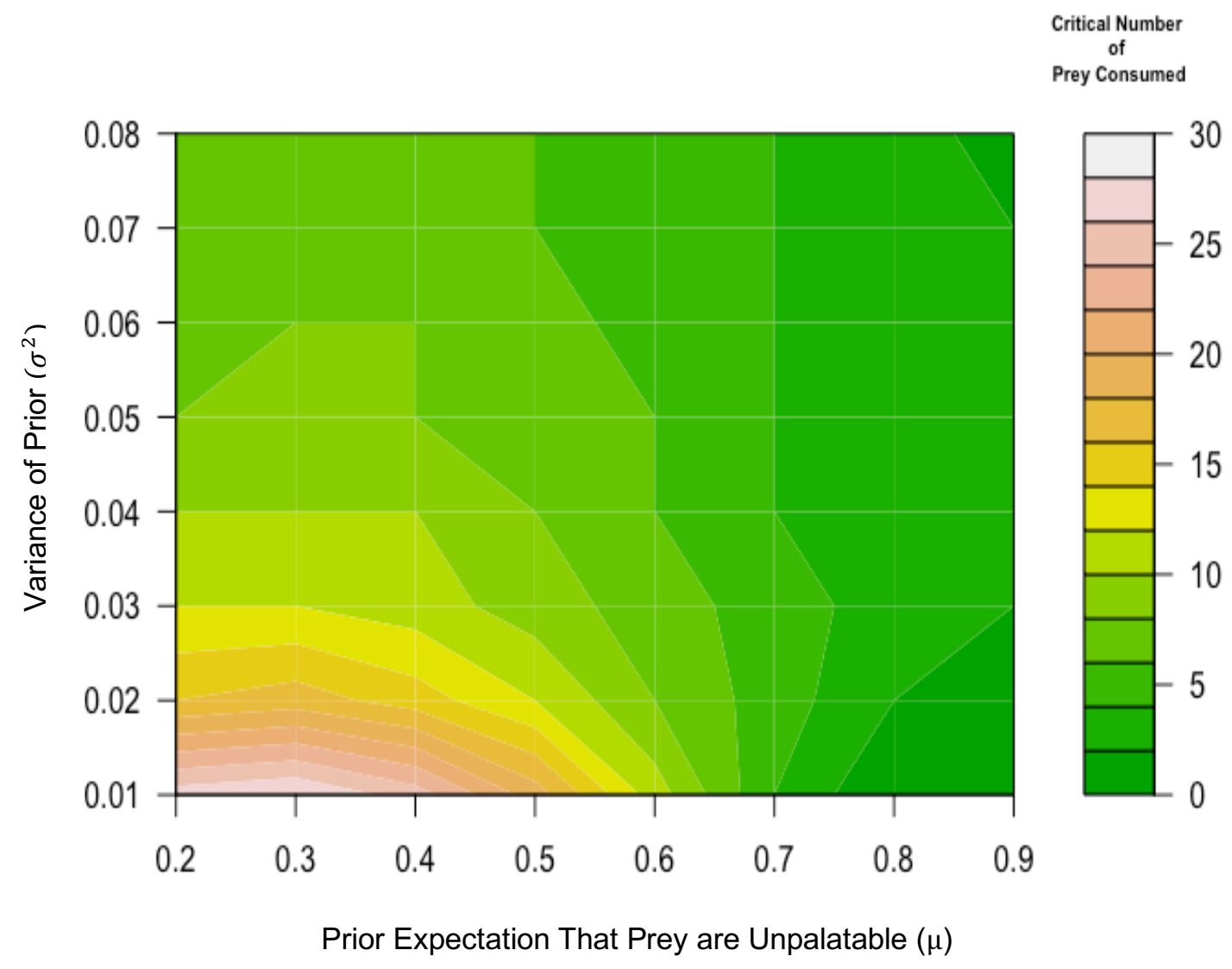

Figure 3.2 The critical number of unpalatable prey consumed by a predator as a function of the predator's Bayesian prior expectation $(\alpha)$ and variance $\left(\sigma^{2}\right)$ that the prey are unpalatable. The cost of attacking an unrewarding prey was set at 1, and the benefit of attacking a rewarding prey was set at 2 in the model. 
Collectively, the results from my model indicate that a predator's previous knowledge and/or belief concerning the profitability of an unfamiliar prey, as well as the certainty the predator has in its belief, will affect its decision to attack, and whether to continue attacking, an unfamiliar prey type. Knowing this, we can use this model to understand the implications of the optimal sampling strategy for a natural predator faced with unfamiliar prey, including in particular implications for the evolution of aposematism, where chemically defended prey signal their unprofitability to predators using conspicuous warning coloration (Poulton, 1890). This is done by recognizing its consequences for prey mortality. Figure 3.1 shows that a predator with the previous belief that unfamiliar prey are unpalatable will reduce its attack rate and will completely reject the unfamiliar prey after fewer attacks, a result supported by Sherratt (2011). Therefore, if a predator begins with the expectation that brightly coloured prey are unprofitable, it should reject aposematic prey sooner because it conforms to its initial expectation. Consequently, this would reduce the attack rate on aposematic prey and overall reduce prey mortality, providing support that conspicuous warning signals favour prey survival. This result has strong empirical support; for example, studies have shown that conspicuous warning signals cause predators to be cautious and to restrain their handling of aposematic prey (Sillén-Tullberg, 1985; Kelly \& Marples, 2004), as well as fast track predator-avoidance learning (Gittleman \& Harvey, 1980; Gittleman et al.,1980 ; Roper \& Wistow,1986; Lindström et al., 2001), together demonstrating that conspicuous warning signals favour the survival of chemically defended prey. In addition, we must also consider that the certainty a predator has in its expectation also affects the optimal sampling strategy. Therefore, if a predator begins with the expectation that unfamiliar prey are unprofitable, it should reject chemically defended prey types sooner as its certainty in its initial expectation increases (Figure 3.2). This suggests that if a predator is 
confident that aposematic prey are unprofitable, then the survival of aposematic prey will be further favoured. However, this hypothesis has yet to be tested empirically.

Lastly, my final aim was to compare the optimal sampling strategy yielded by my model to empirical data, so as to ascertain whether natural predators solve a binary food choice problem (exploration-exploitation trade-off) as would be expected of Bayesian learners (i.e. following the optimal sampling rules generated by my Bayesian model). To do this, I compared the optimal sampling strategy identified above by my model to the results from of my previous binary food choice experiment using Jamaican field crickets, Gryllus assimilis (Chapter 2). As a reminder, in the previous study, I tested the ability of crickets to learn a novel binary food choice. In that study, each cricket was presented with a total of 14 binary food choices and each choice consisted of a rewarding and unrewarding novel prey type. Overall, the results from this experiment (Chapter 2) showed that crickets were capable of learning a binary food choice and food preference. Regarding my Bayesian model here, to more closely simulate the conditions under which the crickets were tested, I made several assumptions and adjustments to my model. First, for model simulation, I assumed the predator was now faced with a binary food choice between two different prey types, and that it was capable of discriminating between the two prey. Second, I assumed that one of the two prey types was unpalatable, and that the other prey type was palatable and would always be attacked if encountered by the predator. Third, I assumed the predator adopted Bayesian learning, and began with a prior expectation of 0.5 that unfamiliar prey are unpalatable (specifically Beta $(1,1))$. Lastly, I assumed that when a predator simultaneously encountered a pair of prey, the probability that the predator would choose one prey type (palatable) or the other prey type (unpalatable) follows one of two rules. The first rule 
assumed that the probability of a predator choosing a prey was 0.5 . In other words, I assumed that $50 \%$ of the time the predator would choose the unpalatable prey type (Figure 3.3). The second rule assumed that the probability of a predator choosing a prey was based on its preference, or its prior expectation that the prey was unpalatable (Bayesian priors) (Figure 3.4). To solve for the optimal sampling strategy, I set (i) the total number of a given prey type to 14 to mimic the 14 binary choices presented to the crickets in any given experimental learning trial, (ii) the cost of attacking an unrewarding prey to 0.5 to account for the loss of energy and time a cricket incurred by attacking an unrewarding prey, and (iii) the benefit of attacking a rewarding prey to 1 to account for the benefit to the cricket for attacking and consuming a palatable food source. Note, once a predator visits a binary choice, it cannot revisit that choice at a later time.

Figures 3.3 and 3.4 compare the optimal sampling strategy to the empirical learning strategies used by crickets in my previous experiment (Chapter 2). Figure 3.3 shows the optimal sampling strategy for a predator following the assumption that the predator chooses the palatable prey type $50 \%$ of the time (at random), and Figure 3.4 shows the optimal sampling strategy for a predator following the assumption that the predator chooses the palatable prey based on its prior expectation (Bayesian priors). First, it is clear that the optimal sampling strategies differ from the empirical learning strategies used by crickets. Under both assumptions, the optimal sampling strategy is for the predator to begin by sampling the unpalatable prey (this initial attack rate will reflect the predator's preference), and with experience eventually reject the unpalatable prey type completely and instead exploit the palatable prey type for the remaining pairs encountered. Contrary to my model's predictions, my empirical results show that the crickets exhibited little exploration and instead started with, and maintained, a steady attack rate (much higher than 0.5) 
on the rewarding prey type across all pairs encountered. Second, it is clear that the two optimal sampling strategies differ from one another. Largely, by comparing Figures 3.3 and 3.4, it is clear that a predator's prior beliefs (Bayesian priors) have an effect on the optimal sampling strategy. Figure 3.4 shows the optimal sampling strategy of a predator who chooses the palatable prey from its prior expectation. Unlike in Figure 3.3, where the predator continues to choose the palatable prey at random $(50 \%)$, in Figure 3.4 the predator continually updates its expectation as it gains new evidence. Here, we can see that a predator's prior expectation affects the optimal sampling strategy. A predator with the prior belief that unfamiliar prey are unpalatable will begin with a higher initial attack rate on palatable prey, and will completely avoid attacking the unpalatable prey sooner because this conforms to their expectation. For example, a predator starting with a prior expectation of $0.67(\operatorname{Beta}(2,1))$, will begin with an attack rate of approximately 0.67 on palatable prey, and will soon reach the optimal switch point after encountering only 10 prey pairs (Figure $3.4 \mathrm{C}$.). Whereas, a predator starting with a lower expectation of $0.5(\operatorname{Beta}(1,1))$ or even $0.33(\operatorname{Beta}(1,2))$, will begin with a lower initial attack rate of around 0.5 and 0.33 respectively on palatable prey, and will require more evidence (12 and 14 prey pairs respectively) before reaching the optimal switching point, because this opposes their prior expectation (Figure 3.4 A., B.). Again, this confirms our earlier finding that a predator's prior beliefs (Bayesian priors) have an effect on the optimal sampling strategy. 


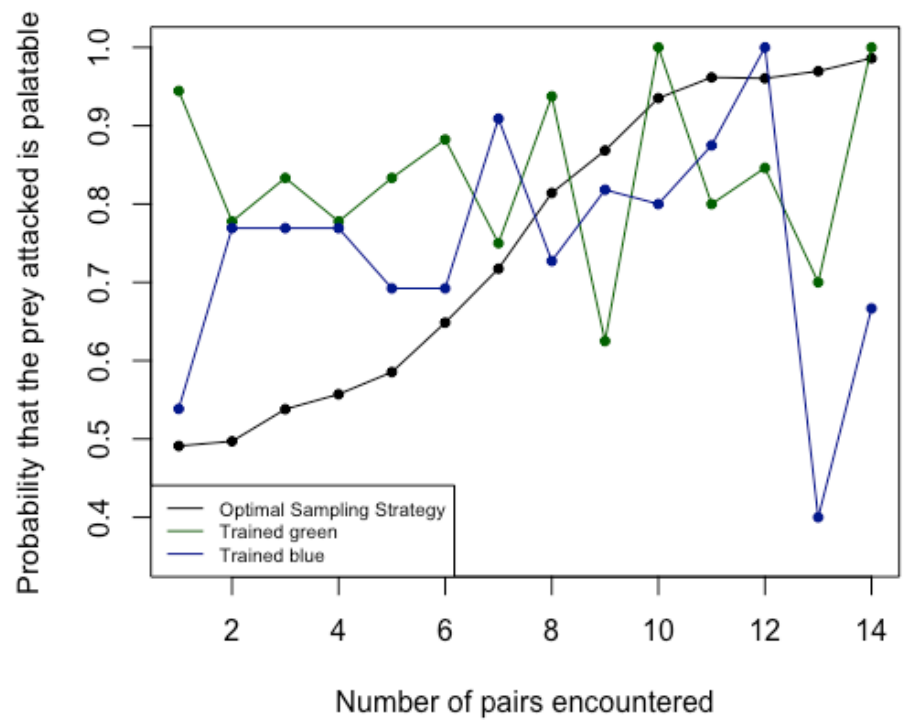

Figure 3.3. Comparison of the optimal sampling strategy to empirical learning data in making a novel binary food choice. Here the optimal sampling strategy follows the assumption that a predator chooses a prey type at random, or a probability of 0.5 . The empirical data is taken from Trial 1 of my previous experiment using Jamaican field crickets. 
A.

$\alpha=1, \beta=2$

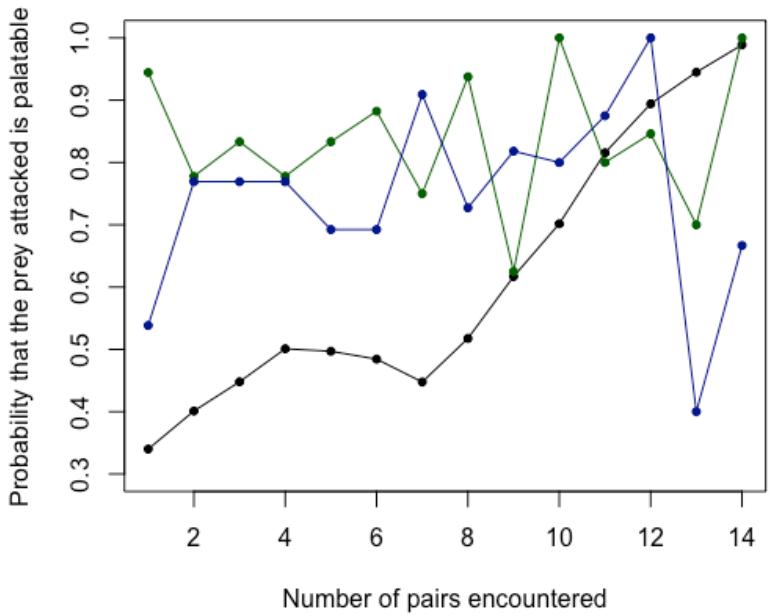

- Optimal Sampling Strategy

- Trained green

- Trained blue

B.

$\alpha=1, \beta=1$

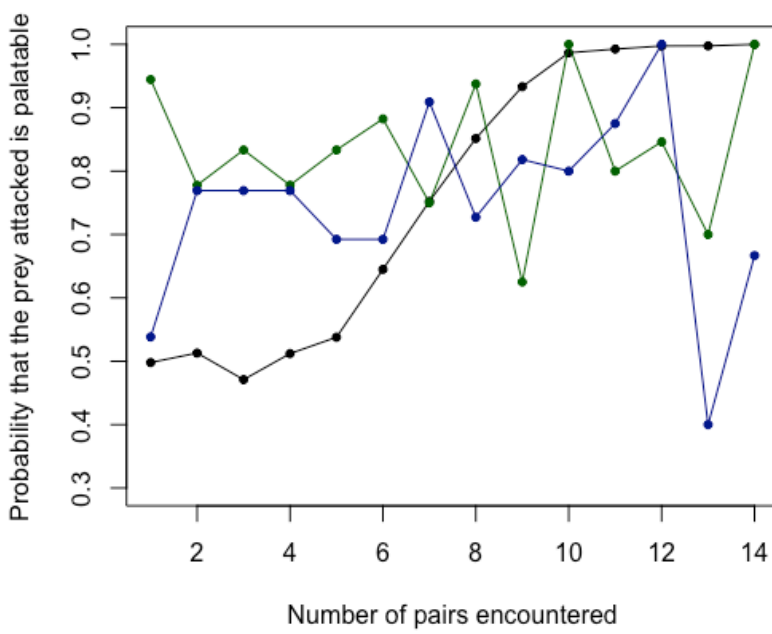

$\alpha=2, \beta=1$

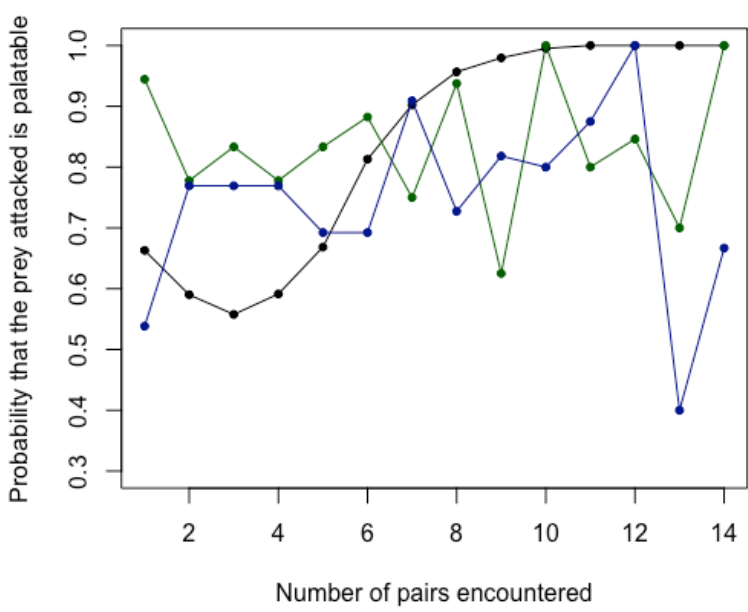

— Optimal Sampling Strategy Trained green

Trained blue

Figure 3.4. Comparison of the optimal sampling strategy to empirical learning data in making a novel binary food choice. Here the optimal sampling strategy follows the assumption that a predator chooses a prey type based from its prior expectation (Bayesian priors). The empirical data is taken from Trial 1 of my previous experiment using Jamaican field crickets. 
As previously mentioned, it is evident that my empirical results (Chapter 2) with crickets do not conform to those predicted by my Bayesian model following either assumption (Figures 3.3 and 3.4). Looking at Figures 3.3 and 3.4, a major concern is the crickets' immediate (initial) high attack probability on palatable prey. From the very start of my experiment, crickets exhibited an attack probability of approximately $75 \%$ on palatable prey (averaged over both colour groups), rather than an expected attack rate of around $50 \%$. This initial attack rate is even higher (i.e. approximately $95 \%$ ) for crickets trained with the rewarding prey coloured green. A possible explanation for this result is that crickets used some form of visual or olfactory cue(s) to identify the rewarding prey type and successfully made the binary food choice without any experience. This would explain why crickets began with such a high attack probability on the palatable prey and maintained it throughout the trial. If this assumption is correct, and crickets used sensory cues to discriminate the rewarding prey, then my model (following either assumption) is not a good reflection of the cricket's prey attack and learning behaviour. Under this assumption, this provides a possible explanation as to why my empirical results (Chapter 2) with crickets did not conform to those predicted by my Bayesian model (Figures 3.3 and 3.4). In addition, it is important to note that my learning model does not account for the exact conditions of my learning experiment with crickets. Largely, my model assumes, under one condition, that a predator chooses the rewarding prey type at random $(50 \%)$ and, under a second condition, that a predator chooses the rewarding prey type based from its prior expectation. Contrary to these assumptions, in my learning experiment (Chapter 2), the crickets seemed to consistently and correctly discriminate and choose the rewarding prey type from the pair. Together, this provides a second possible explanation as to why my empirical results (Chapter 2) with crickets did not conform to those predicted by my Bayesian model (Figures 3.3 and 3.4). 
As a potential next step, I would be interested in modifying my learning model as a twoarmed bandit in which both arms are unknown, and solving for the optimal sampling strategy. On one hand, such a modified model would provide a solution to predator's choosing a prey type at random (50\%). However, on the other hand, the model would cause a slight problem, such that crickets would be able to revisit a prey pair that they have previously visited for more information. In this case, modifying my current Bayesian model would require a different experimental protocol than the one I adopted for my previous experiment (Chapter 2), namely that, once a cricket has visited a pair of prey items (and did not attack), this pair subsequently remains available for a potential future predator encounter/visit. Overall, such a modified model would provide a better representation of my previous learning experiment with crickets, and hopefully provide deeper insight into the cricket prey attack and learning behaviours observed in Chapter 2.

In addition, using this model, I could compare the optimal sampling strategy to other empirical datasets to assess whether natural predators behave according to the optimal sampling strategy when faced with a novel food choice consisting of two unfamiliar prey. 


\section{CHAPTER 4}

General Discussion 


\section{Overview of salient results}

I experimentally demonstrated that laboratory-reared, adult Jamaican field crickets are capable of learning a binary food choice (Chapter 2). In this experiment, crickets were trained with two prey types, a rewarding prey consisting of a piece of Gala apple and an unrewarding prey consisting of a piece of fun foam, dyed either blue or green. Individual test animals underwent a total of 5 consecutive days of learning trials, and were presented with 14 unfamiliar binary food choices within each trial. As trials progressed, crickets increasingly made more correct choices (rewarding prey), demonstrating a learned food preference across trials. Note that learning cannot be entirely attributed to colour, potential alternative cues must be considered such as, olfactory cues, as well as alternative visual cues such as, symbols, texture, and/or contrast. To my knowledge, this is the first test of crickets using visual cues (colour in this case) to learn a food preference. Previous research has shown that crickets are capable of visual discrimination and learning (Campan \& Lacoste, 1973) and of learning a food preference, as well responding to a binary choice using olfactory cues (Matsumoto \& Mizunami 2000, 2004). Notably, my results provide the first evidence of crickets (i) demonstrating an ability to use visual discriminatory cues (colour) to learn a food preference and (ii) successfully responding to a binary food choice.

Interestingly, in my experiment (Chapter 2), the colour of the prey had a significant effect on the proportion of correct (rewarding prey) choices made. Crickets trained with the rewarding prey coloured green made significantly more correct choices compared to the crickets trained with the rewarding prey coloured blue. There are several plausible explanations for this finding. First, it could be that crickets possess an innate colour preference for the colour green. In nature, various hues of green and brown are common in the natural habitats of crickets (Prudic et al., 
2006) and these hues are commonly associated with prey palatability. Therefore, it is possible that crickets possess an innate preference for green coloured prey, because it is a familiar colour often associated with palatable and harmless prey. Evidence of innate colour preferences have been widely documented in insects. However, most research has focused on flower-visiting insects, including Papillonidae (Ilse \& Vaidya, 1956; Weiss, 1997), Pieridae (Traynier, 1984; Scherer \& Kolb, 1987), Nymphalidae (Swihart \& Swihart, 1970), Sphingidae (Kebler, 1996; Goyret et al., 2008), and Apidae (Giurfa et al., 1995), with only a few studies demonstrating innate colour preferences in orthopteran species (Bernays \& Wrubel, 1985; Holliday \& Holliday, 1995; Behmer, 2005). In the current study, I came close to testing for an innate colour preference in crickets, but did not do so specifically. To confirm such an assumption and to directly test for an innate colour preference, both novel colour cues used in my experiment (green and blue) would have to be associated with an equal reward. However, if crickets did show an innate preference for the colour green, it would explain why those crickets trained with the rewarding prey coloured green made significantly more correct choices compared to crickets trained with the rewarding prey coloured blue. Conversely, as an alternative explanation, it could be that the reluctance of crickets to associate the colour blue with a reward could have been influenced by an innate bias towards warning coloration. This proposition is supported by previous research (e.g. Gittleman \& Harvey, 1980; Gittleman et al., 1980). If crickets in my experiment possessed an innate avoidance of aposematic colours, then they may have assessed the strongly contrasting blue prey to be aposematic and thus advertising toxicity or unprofitability. This could explain why crickets trained with the rewarding prey coloured blue made significantly fewer correct choices compared to the crickets trained with the rewarding prey coloured green. 
Furthermore, the effect of colour on the proportion of correct choices made by crickets could also be caused by differences in the cricket's visual sensory system. For example, it is possible that crickets have developed a higher sensitivity to colour wavelengths detected by green photoreceptors compared to blue photoreceptors, therefore allowing better discrimination of green coloured prey, and consequently a greater proportion of green colour prey attacked.

Lastly, it is possible that the effect of colour on the proportion of correct choices made by crickets is due to differences between the two colours of food dye. First, it is possible that the blue and green food dyes disguised the cues that the crickets used to recognize the rewarding prey type, but to different degrees. In this case, if the blue dye disguised the cues of the rewarding prey (whether visual or olfactory) more heavily compared to the green dye, it would explain why crickets trained with the rewarding prey coloured blue made fewer correct choices. Secondly, it is also possible that the blue and green food dyes possess slightly different ingredients, and as a result the olfactory cues and/or taste of the two coloured prey differed. In this case, if the green dye emitted more attractive olfactory cues or a preferred taste, it could also explain the significant effect of colour on the proportion of rewarding prey attacked.

In Chapter 3, I demonstrated using mathematical modelling that a predator's prior biases (beliefs) concerning the palatability of a novel prey affects its decision to attack, and whether to keep attacking an unfamiliar prey. I formalized a predator's decision to attack a prey item or not as an exploration-exploitation trade-off, and solved for the predator's optimal sampling strategy assuming Bayesian learning. Although the effect of a predator's Bayesian priors on the optimal sampling strategy has previously been confirmed (Sherratt, 2011), to my knowledge, my model 
this is the first to elucidate the influence of a predator's certainty in its belief on the optimal sampling strategy.

The optimal switch point, the point at which a predator should completely avoid attacking unfamiliar prey if they all turn out to be unpalatable, was determined for a predator beginning with a range of set Bayesian priors. A predator starting with the initial belief that unfamiliar prey are unpalatable had a more conserved sampling strategy compared to a predator starting with the initial belief that unfamiliar prey are palatable. The greater the predator's initial mean expectation of unfamiliar prey being unpalatable, the more conserved the optimal switch point. Moreover, the certainty a predator has in its initial belief also affected the optimal sampling strategy. A predator with a high mean expectation that unfamiliar prey are unpalatable, and a high certainty (low variance around the mean) in this expectation, will have a more conserved sampling strategy compared to a predator with a low certainty (high variance around the mean) in its prior expectation. The opposite is true for a predator starting with a low mean expectation that unfamiliar prey are unpalatable. Collectively, these results indicate that a predator's prior beliefs concerning the palatability of unfamiliar prey, and its certainty in its beliefs, affect its decision to attack and whether to keep attacking unfamiliar prey, and overall influence the optimal sampling strategy. These results are important as they demonstrate that a predator's prior belief concerning the profitability of a novel prey, such as an innate preference or a predisposition towards warning coloration, and the strength of that belief, affects the predator's decision to attack all unfamiliar prey, consequently affecting prey selection. 
Lastly, we can use these findings to link the results of my learning model (Chapter 3) to the experimental results from Chapter 2, specifically the effect of colour on the proportion of correct choices made. In Chapter 2, I found that crickets trained with the rewarding prey coloured green made significantly more correct choices (rewarding prey) compared to crickets trained with the rewarding prey coloured blue. One proposed explanation for this result is that crickets might possess an innate bias for green hues, which in turn could underlie a potential innate preference for green coloured prey. According to my learning model (Chapter 3), an innate preference for green coloured prey would suggest that crickets had a positive prior bias concerning the palatability of green coloured prey, and therefore had a lower expectation that green coloured prey were unpalatable. As a result, they attacked more green coloured prey. Alternatively, if the reluctance of crickets to associate the colour blue with a reward was influenced by an innate bias towards warning coloration, then plausibly they may have possessed a negative bias towards the palatability of blue coloured prey and a higher expectation that blue coloured prey were unpalatable, thus resulting in fewer blue coloured prey attacked. Yet, despite my model accounting for these different priors for blue and green coloured prey, it failed to accurately reflect the experimental data presented in Chapter 2. This is evident by comparing the learning data from Chapter 2 to the optimal sampling strategy identified by my Bayesian model in Chapter 3, and observing a clear difference between them. This suggests that an alternative explanation to an innate colour preference/avoidance may explain the observed effect of colour on the proportion of rewarding prey attacked by crickets. For example, it could be instead that the visual acuity of crickets towards green versus blue coloured prey differed, or that the food colouring per se affected the visual or olfactory cues of the two prey types perceived by the crickets. Therefore, it is entirely possible the conditions and assumptions made by my learning 
model did not replicate those of my learning experiment, and consequently do not provide a clear understanding of the experimental data.

\section{Directions for future research}

The experimental work described herein, demonstrating the ability of Jamaican field crickets to learn a novel binary food choice, enhances our understanding of learning behaviour in crickets. My findings show that crickets used visual cues to learn a food preference. This is supported by finding a significant effect of colour on the proportion of correct choices made. However, due to the limitations of my study, I cannot confirm whether this preference was learned entirely by colour or whether other sensory cues, such as other visual cues or olfactory cues, also contributed to crickets learning the observed food preferences. In my current study, I controlled for alternative forms of visual cues by choosing two prey types that shared a similar texture and were the same size, sharing the same dimensions (1 $\mathrm{mm} \times 1 \mathrm{~mm} \times 1 \mathrm{~mm})$. However, despite my efforts, it is possible that the crickets were still able to discriminate between the two prey types using visual cues. Although I controlled for olfactory cues by brushing the unrewarding prey (fun foam) with a piece of Gala apple (rewarding prey) before including it in a trial, it is nonetheless possible that this control was insufficient or ineffective. Brushing the unrewarding prey with Gala apple may not have balanced out the differences in olfactory cues between the two prey types and/or the rewarding and unrewarding prey types (apple and fun foam) may have reacted differentially to the added food dye, creating a difference in olfaction. The use of olfactory cues in insect learning has been characterized for a range of behaviours, including but not limited to host foraging behaviour (Drost et al.,1986;Wackers \& Lewis, 1993), prey differentiation (Matsumoto \& Mizunami, 2000), and plant-animal communication (Papaj \& 
Lewis, 1993; Hansson, 1999; Raguso \& Willis, 2002 and 2005). Given that olfaction is an ecologically important sensory modality in animals in general, I would be interested in testing whether it is also implicated in insects responding to a novel binary food choice. Therefore, as a next step, I would be interested in testing the ability of crickets to learn the same food preference after having (i) completely eliminated and controlled for any/all olfactory cues, (ii) completely eliminated and controlled for any/all visual cues, and (iii) including both visual and olfactory cues. This would allow me to test whether crickets can learn a novel binary food choice using only visual or olfactory cues, or using both visual and olfactory cues simultaneously, while providing insight into whether crickets are capable of using multimodal sensory cues, and whether one sensory modality (vision or olfaction) is relied upon more than the other, in learning to choose among unfamiliar prey items that differ in palatability/profitability.

The theoretical work described herein (Chapter 3), characterizing the optimal sampling strategy for a predator faced with unfamiliar prey, suggests that a predator's previous knowledge and/or prior biases affect its decisions to attack and whether to keep attacking unfamiliar prey. The results of my theoretical model (Chapter 3) can be used to explore further hypotheses for the evolution of prey selection and prey coloration, in particular concerning the evolution of conspicuous coloration and aposematism. Unfortunately, in the current study, this theoretical finding was not fully supported by my empirical data with crickets (Chapter 2). Presently, my learning model describes a situation where a predator is faced with a binary food choice in which the predator is capable of discriminating between the two prey types. In my current Bayesian model of learning, one prey type is unpalatable, and the other is palatable and assumed to always be attacked if chosen. The predator is assumed to choose the palatable prey at random (50\%) or 
based on its prior expectation (Bayesian priors). Under these conditions, my model solves for the predator's optimal sampling strategy. As a next step, I would be interested in modifying my learning model as a two-armed bandit in which both arms are unknown, and solving for the optimal sampling strategy. On one hand, such a modified model would provide a solution to predator's choosing a prey type at random (50\%). However, on the other hand, the model would cause a slight problem, such that crickets would be able to revisit a prey pair that they have previously visited for more information. In this case, modifying my current Bayesian model would require a different experimental protocol than the one I adopted for my previous experiment (Chapter 2), namely that, once a cricket has visited a pair of prey items (and did not attack), this pair subsequently remains available for a potential future predator encounter/visit. I believe that a such a modified model would better represent my empirical work completed in Chapter 2, and thus more likely to provide insights into the learning behaviour of foraging crickets. 


\section{References}

Alcock, J. (2013). The development of behaviour. In: Animal behavior: An evolutionary approach (10th ed.). Sinauer Associates, Sunderland, MA, pp. 349-355.

Alexander, R. D., Marshall, D. C., \& Cooley, J. R. (1997). Evolutionary perspectives on insect mating. In: The evolution of mating systems in insects and arachnids. Choe, J. \& Crespi, B. (eds.), Cambridge University Press, Cambridge, UK, pp. 4-31.

Alloway, T. M. (1972). Learning and memory in insects. Annual Review of Entomology, 17(1), 43-56.

Alonso, J. C., Alonso, J. A., Bautista, L. M., \& Muñoz-Pulido, R. (1995). Patch use in cranes: a field test of optimal foraging predictions. Animal Behaviour, 49(5), 1367-1379.

Aronsson, M., \& Gamberale-Stille, G. (2008). Domestic chicks primarily attend to colour, not pattern, when learning an aposematic coloration. Animal Behaviour, 75(2), 417-423.

Aubier, T. G., \& Sherratt, T. N. (2015). Diversity in Müllerian mimicry: the optimal predator sampling strategy explains both local and regional polymorphism in prey. Evolution, 69(11), 2831-2845.

Behmer, S. T., Belt, C. E., \& Shapiro, M. S. (2005). Variable rewards and discrimination ability in an insect herbivore: what and how does a hungry locust learn?. Journal of Experimental Biology, 208(18), 3463-3473.

Bernays, E. A., \& Wrubel, R. P. (1985). Learning by grasshoppers: association of colour/light intensity with food. Physiological Entomology, 10(4), 359-369.

Bernstein, I. L., Vitiello, M. V., \& Sigmundi, R. A. (1980). Effects of interference stimuli on the acquisition of learned aversions to foods in the rat. Journal of Comparative and Physiological Psychology, 94(5), 921-931.

Berry, D. A., \& Fristedt, B. (1985). Bandit problems: sequential allocation of experiments (Monographs on statistics and applied probability). Cox, D.R, Hinkley, D.V., Rubin, D, \& Silverman, B (Eds.), Chapman and Hall, London, UK, pp.71-87.

Beugnon, G., \& Campan, R. (1989). Homing in the field cricket, Gryllus campestris. Journal of Insect Behavior, 2(2), 187-198.

Beugnon, G. (1986). Learned orientation in landward swimming in the cricket Pteronemobius lineolatus. Behavioural Processes, 12(3), 215-226.

Bolker, B. M. (2008). Probability and stochastic distributions for ecological modelling. In: Ecological models and data in R. Princeton University Press, Princeton, NJ, pp. 103-120. 
Braveman, N. S. (1975). Relative salience of gustatory and visual cues in the formation of poison-based food aversions by guinea pigs (Cavia porcellus). Behavioral Biology, 14(2), 189199.

Brown, G. E., \& Godin, J.-G. J. (1999). Who dares, learns: chemical inspection behaviour and acquired predator recognition in a characin fish. Animal Behaviour, 57(2), 475-481.

Burghardt, G. M., Wilcoxon, H. C., \& Czaplicki, J. A. (1973). Conditioning in garter snakes: Aversion to palatable prey induced by delayed illness. Animal Learning and Behavior, 1(4), 317 320.

Burt de Perera, T., \& Guilford, T. C. (2008). Rapid learning of shelter position in an intertidal fish, the shanny Lipophrys pholis L. Journal of Fish Biology, 72(6), 1386-1392.

Cammaerts, M. C. (2008). Visual discrimination of cues differing as for their number of elements, their shape or their orientation, by the ant Myrmica sabuleti. Biologia, 63(6), 11691180 .

Campan, R., \& Lehrer, M. (2002). Discrimination of closed shapes by two species of bee, Apis mellifera and Megachile rotundata. Journal of Experimental Biology, 205(4), 559-572.

Capaldi, E. A., Smith, A. D., Osborne, J. L., Fahrbach, S. E., Farris, S. M., Reynolds, D. R., Edwards, A.S., Martin, A., Robinson, G.E., Poppy, G.M., \& Riley, J. R. (2000). Ontogeny of orientation flight in the honeybee revealed by harmonic radar. Nature, 403(6769), 537.

Capretta, P. J. (1961). An experimental modification of food preference in chickens. Journal of Comparative and Physiological Psychology, 54(3), 238-242.

Cartwright, B. A., \& Collett, T. S. (1982). How honey bees use landmarks to guide their return to a food source. Nature, 295(5850), 560-564.

Chivers, D. P., \& Smith, R. J. F. (1994). Fathead minnows, Pimephales promelas, acquire predator recognition when alarm substance is associated with the sight of unfamiliar fish. Animal Behaviour, 48(3), 597-605.

Chivers, D. P., \& Smith, R. J. F. (1998). Chemical alarm signalling in aquatic predator-prey systems: a review and prospectus. Écoscience, 5(3), 338-352.

Clark, C. W., \& Mangel, M. (2000). Forward iteration and Monte Carlo simulation. In: Dynamic state variable models in ecology: methods and applications. Oxford University Press, New York, NY, pp. 54-57.

Collett, T. S., Dillmann, E., Giger, A., \& Wehner, R. (1992). Visual landmarks and route following in desert ants. Journal of Comparative Physiology A, 170(4), 435-442. 
Curio, E., Ernst, U., \& Vieth, W. (1978). Cultural transmission of enemy recognition: one function of mobbing. Science, 202(4370), 899-901.

Czaplicki, J. A., Borrebach, D. E., \& Wilcoxon, H. C. (1976). Stimulus generalization of an illness-induced aversion to different intensities of colored water in Japanese quail. Animal Learning \& Behavior, 4(1), 45-48.

Dall, S. R., Giraldeau, L. A., Olsson, O., McNamara, J. M., \& Stephens, D. W. (2005). Information and its use by animals in evolutionary ecology. Trends in Ecology \& Evolution, 20(4), 187-193.

Darmaillacq, A. S., Dickel, L., Chichery, M. P., Agin, V., \& Chichery, R. (2004). Rapid taste aversion learning in adult cuttlefish, Sepia officinalis. Animal Behaviour, 68(6), 1291-1298.

Darwin, C. (1877). The effects of cross- and self- fertilization in the vegetable kingdom. In: The different forms of flowers on plants of the same species. Murray, London, pp. 425-426.

Davis, R. L. (2005). Olfactory memory formation in Drosophila: from molecular to systems neuroscience. Annual Review of Neuroscience. 28, 275-302.

de Ibarra, N. H., \& Giurfa, M. (2003). Discrimination of closed coloured shapes by honeybees requires only contrast to the long wavelength receptor type. Animal Behaviour, 66(5), 903-910.

Drea, C. M. (2006). Studying primate learning in group contexts: Tests of social foraging, response to novelty, and cooperative problem solving. Methods, 38(3), 162-177.

Drost, Y. C., Lewis, W. J., Zanen, P. O., \& Keller, M. A. (1986). Beneficial arthropod behavior mediated by airborne semiochemicals. Journal of Chemical Ecology, 12(6), 1247-1262.

Dukas, R. (Ed.). (1998). Cognitive ecology: the evolutionary ecology of information processing and decision making. University of Chicago Press.

Dukas, R. (1999). Ecological relevance of associative learning in fruit fly larvae. Behavioral Ecology and Sociobiology, 45(3-4), 195-200.

Dukas, R. (2005a). Experience improves courtship in male fruit flies. Animal Behaviour, 69(5), 1203-1209.

Dukas, R. (2005b). Learning affects mate choice in female fruit flies. Behavioral Ecology, 16(4), 800-804.

Dukas, R. (2008). Evolutionary biology of insect learning. Annual Review of Entomology, 53, 145-160.

Ellison, A. M. (1996). An introduction to Bayesian inference for ecological research and environmental decision-making. Ecological applications, 6(4), 1036-1046. 
Ferrari, M. C., Gonzalo, A., Messier, F., \& Chivers, D. P. (2007). Generalization of learned predator recognition: an experimental test and framework for future studies. Proceedings of the Royal Society of London B: Biological Sciences, 274(1620), 1853-1859.

Ferrari, M. C., Trowell, J. J., Brown, G. E., \& Chivers, D. P. (2005). The role of learning in the development of threat-sensitive predator avoidance by fathead minnows. Animal Behaviour, 70(4), 777-784.

Fouks, B., \& Lattorff, H. M. G. (2011). Recognition and avoidance of contaminated flowers by foraging bumblebees (Bombus terrestris). PLoS One, 6(10), e26328.

Garcia, J., \& Koelling, R. A. (1966). Relation of cue to consequence in avoidance learning. Psychonomic Science, 4(1), 123-124.

Gelperin, A. (1975). Rapid food-aversion learning by a terrestrial mollusk. Science, 189(4202), 567-570.

Gibson, E. J. (1969). Principles of perceptual learning and development. Appleton-CenturyCrofts, East Norwalk, CT.

Gittleman, J. L., \& Harvey, P. H. (1980). Why are distasteful prey not cryptic?. Nature, 286(5769), 149-150.

Gittleman, J. L., Harvey, P. H., \& Greenwood, P. J. (1980). The evolution of conspicuous coloration: some experiments in bad taste. Animal Behaviour, 28(3), 897-899.

Giurfa, M., Nunez, J., Chittka, L., \& Menzel, R. (1995). Colour preferences of flower-naive honeybees. Journal of Comparative Physiology A, 177(3), 247-259.

Goyret, J., Pfaff, M., Raguso, R. A., \& Kelber, A. (2008). Why do Manduca sexta feed from white flowers? Innate and learnt colour preferences in a hawkmoth. Naturwissenschaften, 95(6), 569-576.

Griffin, A. S., Evans, C. S., \& Blumstein, D. T. (2001). Learning specificity in acquired predator recognition. Animal Behaviour, 62(3), 577-589.

Hale, R. J., \& Bailey, W. J. (2004). Homing behaviour of juvenile Australian raspy crickets (Orthoptera: Gryllacrididae). Physiological Entomology, 29(5), 426-435.

Harrison, S. J., Raubenheimer, D., Simpson, S. J., Godin, J. G. J., \& Bertram, S. M. (2014). Towards a synthesis of frameworks in nutritional ecology: interacting effects of protein, carbohydrate and phosphorus on field cricket fitness. Proceedings of the Royal Society B: Biological Sciences, 281(1792), 20140539.

Healy, S. (Ed.). (1998). Spatial representation in animals. Oxford University Press, New York, NY. 
Holliday, J. L., \& Holliday, N. J. (1995). Changes in learning ability and mechanism during development of grasshopper nymphs, Melanoplus bivittatus. Physiological Entomology, 20(2), 109-116.

Huber, F., Moore, T. E., \& Loher, W. (Eds.). (1989). Cricket behavior and Neurobiology. Cornell University Press, Ithaca, NY.

Ilse, D., \& Vaidya, V. G. (1956). Spontaneous feeding response to colours in Papilio demoleus L. Proceedings of the Indian Academy of Sciences-Section B, 43(1), 23-31.

Irwin, D. E., \& Price, T. (1999). Sexual imprinting, learning and speciation. Heredity, 82(4), 347-354.

Joron, M. (2009). Aposematic coloration. In: Encyclopedia of Insects. Resh, V.H., \& Cardé, R.T. (eds.), Academic Press, New York, NY, pp. 33-38.

Keasar, T., Rashkovich, E., Cohen, D., \& Shmida, A. (2002). Bees in two-armed bandit situations: foraging choices and possible decision mechanisms. Behavioral Ecology, 13(6), 757765 .

Kelber, A. (1996). Colour learning in the hawkmoth Macroglossum stellatarum. Journal of Experimental Biology, 199(5), 1127-1131.

Kelley, J. L., \& Magurran, A. E. (2003). Learned predator recognition and antipredator responses in fishes. Fish and Fisheries, 4(3), 216-226.

Kelly, D. J., \& Marples, N. M. (2004). The effects of novel odour and colour cues on food acceptance by the zebra finch, Taeniopygia guttata. Animal Behaviour, 68(5), 1049-1054.

Kendrick, K. M., Hinton, M. R., Atkins, K., Haupt, M. A. \& Skinner, J. D. (1998).Mothers determine sexual preferences. Nature, 395: 229-230.

Koops, M., \& Abrahams, M. (2003). Integrating the roles of information and competitive ability on the spatial distribution of social foragers. The American Naturalist, 161(4), 586-600.

Krebs, J. R., Kacelnik, A., \& Taylor, P. (1978). Test of optimal sampling by foraging great tits. Nature, 275(5675), 27-31.

Labhart, T., Baumann, F., \& Bernard, G. D. (2009). Specialized ommatidia of the polarizationsensitive dorsal rim area in the eye of monarch butterflies have non-functional reflecting tapeta. Cell and Tissue Research, 338(3), 391.

Labhart, T., Hodel, B., \& Valenzuela, I. (1984). The physiology of the cricket's compound eye with particular reference to the anatomically specialized dorsal rim area. Journal of Comparative Physiology A, 155(3), 289-296. 
Lee, Y., Poudel, S., Kim, Y., Thakur, D., \& Montell, C. (2018). Calcium taste avoidance in Drosophila. Neuron, 97(1), 67-74.

Lindström, L., Alatalo, R. V., Lyytinen, A., \& Mappes, J. (2001). Predator experience on cryptic prey affects the survival of conspicuous aposematic prey. Proceedings of the Royal Society of London. Series B: Biological Sciences, 268(1465), 357-361.

Lindström, L., Alatalo, R. V., \& Mappes, J. (1999). Reactions of hand-reared and wild-caught predators toward warningly colored, gregarious, and conspicuous prey. Behavioral Ecology, 10(3), 317-322.

Lindström, L., Alatalo, R. V., Mappes, J., Riipi, M., \& Vertainen, L. (1999). Can aposematic signals evolve by gradual change? Nature, 397(6716), 249-251.

Lindström, L., Rowe, C., \& Guilford, T. (2001). Pyrazine odour makes visually conspicuous prey aversive. Proceedings of the Royal Society of London. Series B: Biological Sciences, 268(1463), 159-162.

Little, E. E. (1977). Conditioned aversion to amino acid flavors in the catfish, Ictalurus punctatus. Physiology and Behavior, 19(6), 743-747.

Luttbeg, B. (1996). A comparative Bayes tactic for mate assessment and choice. Behavioral Ecology, 7(4), 451-460.

Luttbeg, B., \& Warner, R. R. (1999). Reproductive decision-making by female peacock wrasses: flexible versus fixed behavioral rules in variable environments. Behavioral Ecology, 10(6), 666674.

MacKay, B. (1974). Conditioned food aversion produced by toxicosis in Atlantic cod. Behavioral Biology, 12(3), 347-355.

Mackay, B. (1977). Visual and flavor cues in toxicosis conditioning of codfish. Behavioral Biology, 19(1), 87-97.

Mangel, M. (1990). Dynamic information in uncertain and changing worlds. Journal of Theoretical Biology, 146(3), 317-332.

Mangel, M., \& Clark, C. W. (1988). Behavioural observations and forwards iteration. In: Dynamic modeling in behavioral ecology. Princeton University Press, Princeton, NJ, pp. 76-79.

Manteifel, Y. B., \& Karelina, M. A. (1996). Conditioned food aversion in the goldfish, Carassius auratus. Comparative Biochemistry and Physiology Part A: Physiology, 115(1), 31-35.

Mason, J. R., \& Reidinger Jr, R. F. (1983). Generalization of and effects of pre-exposure on color-avoidance learning by red-winged blackbirds (Agelaius phoeniceus). The Auk, 100(2), 461468. 
Mastrota, N. F., \& Mench, J. A. (1995). Colour avoidance in northern bobwhites: effects of age, sex and previous experience. Animal Behaviour, 50(2), 519-526.

Mathis, A., \& Smith, R. J. F. (1993). Fathead minnows, Pimephales promelas, learn to recognize northern pike, Esox lucius, as predators on the basis of chemical stimuli from minnows in the pike's diet. Animal Behaviour, 46(4), 645-656.

Matsumoto, Y., \& Mizunami, M. (2000). Olfactory learning in the cricket Gryllus bimaculatus. Journal of Experimental Biology, 203(17), 2581-2588.

Matsumoto, Y., \& Mizunami, M. (2004). Context-dependent olfactory learning in an insect. Learning and Memory, 11(3), 288-293.

Mayr, E. (1974). Behavior programs and evolutionary strategies. American Scientist, 62(6), 650659.

McLeman, M. A., Pratt, S. C., \& Franks, N. R. (2002). Navigation using visual landmarks by the ant Leptothorax albipennis. Insectes Sociaux, 49(3), 203-208.

McNamara, J., \& Houston, A. (1980). The application of statistical decision theory to animal behaviour. Journal of Theoretical Biology, 85(4), 673-690.

Menzel, R., Erber, J., \& Masuhr, T. (1974). Learning and memory in the honeybee. In: Experimental Analysis of Insect Behavior, L. Barton-Browne (ed.), Springer-Verlag, Berlin, pp. 195-217.

Mirza, R. S., Ferrari, M. C., Kiesecker, J. M., \& Chivers, D. P. (2006). Responses of American toad tadpoles to predation cues: behavioural response thresholds, threat-sensitivity and acquired predation recognition. Behaviour, 143(7), 877-889.

Olsson, O., \& Holmgren, N. M. (1998). The survival-rate-maximizing policy for Bayesian foragers: wait for good news. Behavioral Ecology, 9(4), 345-353.

Olsson, O., Wiktander, U., Holmgren, N. M., \& Nilsson, S. G. (1999). Gaining ecological information about Bayesian foragers through their behaviour. II. A field test with woodpeckers. Oikos, 87(2), 264-276.

Papaj, D. R., \& Lewis, A. C. (Eds.). (2012). Insect Learning: Ecology and Evolutionary Perspectives. Springer Science \& Business Media, New York, NY.

Paradise, C. J., \& Stamp, N. E. (1991). Prey recognition time of praying mantids (Dictyoptera: Mantidae) and consequent survivorship of unpalatable prey (Hemiptera: Lygaeidae). Journal of Insect Behavior, 4(3), 265-273.

Pearce, J. M. (2008). Animal learning and cognition: an introduction (3 ${ }^{\text {rd }}$ ed.). Psychology Press, New York, NY. 
Pegram, K. V., Nahm, A. C., Rutowski, R. L., \& Todd, S. (2013). Warning color changes in response to food deprivation in the pipevine swallowtail butterfly, Battus philenor. Journal of insect science, 13(1).

Plowright, C. M. S., \& Plowright, R. C. (1987). Oversampling by great tits? A critique of Krebs, Kacelnik, and Taylor's (1978) "Test of optimal sampling by great tits". Canadian Journal of Zoology, 65(5), 1282-1283.

Poulton, E. B. (1890). Warning colours. In: The colours of animals: their meaning and use: especially considered in the case of insects: with chromolithographic frontispiece and sixty-six figures in text. K. Paul, Trench (ed.), Trübner \& Company, London, pp.21-22.

Pratt, S. C., \& Sumpter, D. J. (2006). A tunable algorithm for collective decisionmaking. Proceedings of the National Academy of Sciences USA, 103(43), 15906-15910.

Prudic, K. L., Skemp, A. K., \& Papaj, D. R. (2006). Aposematic coloration, luminance contrast, and the benefits of conspicuousness. Behavioral Ecology, 18(1), 41-46.

Qvarnstrom, A., Blomgren, V., Wiley, C., \& Svedin, N. (2004). Female collared flycatchers learn to prefer males with an artificial novel ornament. Behavioral Ecology, 15(4), 543-548.

Raguso, R. A., \& Willis, M. A. (2002). Synergy between visual and olfactory cues in nectar feeding by nave hawkmoths, Manduca sexta. Animal Behaviour, 64(5), 685-695.

Raguso, R. A., \& Willis, M. A. (2005). Synergy between visual and olfactory cues in nectar feeding by wild hawkmoths, Manduca sexta. Animal Behaviour, 69(2), 407-418.

Reid, C. R., MacDonald, H., Mann, R. P., Marshall, J. A., Latty, T., \& Garnier, S. (2016). Decision-making without a brain: how an amoeboid organism solves the two-armed bandit. Journal of The Royal Society Interface, 13(119), 20160030.

Rochette, R., Dill, L. M., \& Himmelman, J. H. (1997). A field test of threat sensitivity in a marine gastropod. Animal Behaviour, 54(5), 1053-1062.

Roper, T. J., \& Wistow, R. (1986). Aposematic coloration and avoidance learning in chicks. The Quarterly Journal of Experimental Psychology Section B, 38(2b), 141-149.

Sahley, C., Gelperin, A., \& Rudy, J. W. (1981). One-trial associative learning modifies food odor preferences of a terrestrial mollusc. Proceedings of the National Academy of Sciences USA, $78(1), 640-642$.

Salzen, E. A., Lily, R. E., \& McKeown, J. R. (1971). Colour preference and imprinting in domestic chicks. Animal Behaviour, 19(3), 542-547.

Scherer, C., \& Kolb, G. (1987). Behavioral experiments on the visual processing of color stimuli in Pieris brassicae L. (Lepidoptera). Journal of Comparative Physiology A, 160(5), 645-656. 
Schmidt, V., \& Schaefer, H. M. (2004). Unlearned preference for red may facilitate recognition of palatable food in young omnivorous birds. Evolutionary Ecology Research, 6(6), 919-925.

Schuler, W., \& Hesse, E. (1985). On the function of warning coloration: a black and yellow pattern inhibits prey-attack by naive domestic chicks. Behavioral Ecology and Sociobiology, 16(3), 249-255.

Schuler, W., \& Roper, T. J. (1992). Responses to warning coloration in avian predators. In: Advances in the Study of Behaviour, Academic Press, San Diego, CA, pp.111-146.

Shelly, T. E. \& Bailey, W. J. (1992). Experimental manipulation of mate choice by male katydids: the effect of female encounter rate. Behavioural Ecology and Sociobiology, 30(3-4), 277-282.

Sherratt, T. N. (2011). The optimal sampling strategy for unfamiliar prey. Evolution, 65(7), 2014-2025.

Shettleworth, S. J. (2001). Animal cognition and animal behaviour. Animal Behaviour, 61(2), 277-286.

Sillén-Tullberg, B. (1985). Higher survival of an aposematic than of a cryptic form of a distasteful bug. Oecologia, 67(3), 411-415.

Speed, M. P. (2000). Warning signals, receiver psychology and predator memory. Animal Behaviour, 60(3), 269-278.

Spence, R., \& Smith, C. (2008). Innate and learned colour preference in the zebrafish, Danio rerio. Ethology, 114(6), 582-588.

Swihart, C. A., \& Swihart, S. L. (1970). Colour selection and learned feeding preferences in the butterfly, Heliconius charitonius L. Animal Behaviour, 18, 60-64.

Taylor, L. A., Amin, Z., Maier, E. B., Byrne, K. J., \& Morehouse, N. I. (2015). Flexible color learning in an invertebrate predator: Habronattus jumping spiders can learn to prefer or avoid red during foraging. Behavioral Ecology, 27(2), 520-529.

ten Cate, C., \& Vos, D. R. (1999). Sexual imprinting and evolutionary processes. Advances in the Study of Behavior, 28, 1-31.

Thomas, G., Kacelnik, A., \& Van Der Meulen, J. (1985). The three-spined stickleback and the two-armed bandit. Behaviour, 227-240.

Thorhallsdottir, A. G., Provenza, F. D., \& Balph, D. F. (1987). Food aversion learning in lambs with or without a mother: discrimination, novelty and persistence. Applied Animal Behaviour Science, 18(3), 327-340. 
Traynier, R. M. M. (1984). Associative learning in the ovipositional behaviour of the cabbage butterfly, Pieris rapae. Physiological Entomology, 9(4), 465-472.

Valone, T. (2006). Are animals capable of Bayesian updating? An empirical review. Oikos, 112(2), 252-259.

Valone, T. J., \& Brown, J. S. (1989). Measuring patch assessment abilities of desert granivores. Ecology, 70(6), 1800-1810.

Valone, T. J. (1991). Bayesian and prescient assessment: foraging with pre-harvest information. Animal Behaviour, 41(4), 569-577.

Van Gils, J. A., Schenk, I. W., Bos, O., \& Piersma, T. (2003). Incompletely informed shorebirds that face a digestive constraint maximize net energy gain when exploiting patches. The American Naturalist, 161(5), 777-793.

Vet, L.E.M., \& Lewis, W.J., Carde, R.T. 1995. Parasitoid foraging and learning. In: Chemical Ecology of Insects, Vol. 2, R.T. Carde, \& W.J. Bell (eds.), Chapman and Hall, NY, pp. 65-101.

Vowles, D. M. (1965). Maze learning and visual discrimination in the wood ant (Formica rufa). British Journal of Psychology, 56(1), 15-31.

Wäckers, F. L., \& Lewis, W. J. (1994). Olfactory and visual learning and their combined influence on host site location by the parasitoid Microplitis croceipes (Cresson). Biological Control, 4(2), 105-112.

Wallace, A. R. (1889). Darwinism, an exponent of the theory of natural selection, with some of its applications. Macmillan and Company, London, England, UK.

Weiss, M. R. (1997). Innate colour preferences and flexible colour learning in the pipevine swallowtail. Animal Behaviour, 53(5), 1043-1052.

Wessnitzer, J., Mangan, M., \& Webb, B. (2008). Place memory in crickets. Proceedings of the Royal Society of London B: Biological Sciences, 275(1637), 915-921.

Woody, D. R., \& Mathis, A. (1998). Acquired recognition of chemical stimuli from an unfamiliar predator: associative learning by adult newts, Notophthalmus

viridescens. Copeia, 1998(4), 1027-1031.

Wright, G. A., Mustard, J. A., Simcock, N. K., Ross-Taylor, A. A., McNicholas, L. D., Popescu, A., \& Marion-Poll, F. (2010). Parallel reinforcement pathways for conditioned food aversions in the honeybee. Current Biology, 20(24), 2234-2240.

Zhang, Y., Lu, H., \& Bargmann, C. I. (2005). Pathogenic bacteria induce aversive olfactory learning in Caenorhabditis elegans. Nature, 438(7065), 179-184. 
Zufall, F., Schmitt, M., \& Menzel, R. (1989). Spectral and polarized light sensitivity of photoreceptors in the compound eye of the cricket (Gryllus bimaculatus). Journal of Comparative Physiology A, 164(5), 597-608. 


\section{Appendix A}

\section{Introduction}

The following is an annotated version of the R code used to develop my Bayesian learning model and to simulate a predator adopting Bayesian learning to respond and learn a novel binary food choice.

\section{Fitting the learning model and solving for the critical number of prey a predator should} attack before complete rejection

Here, we assume a predator is faced with a binary food choice, and can discriminate both prey types. We assume only one prey type is unfamiliar to the predator, and that the predator begins with a prior expectation concerning the palatability of the unfamiliar prey, determined by setting the Bayesian priors (alpha $(\alpha)$ and beta $(\beta))$, and uses this expectation in its decision to attack (pref). Let the cost of attacking an unpalatable prey be represented by $c$, the benefit of attacking a palatable prey be represented by $b$, and the total number of available prey be represented by $\operatorname{bigN}$.

\#Set model parameters

pref<- 1

\# 0 for no preference (50\%- attack at random); 1 for preference based on expe rience (Bayesian priors)

alpha<- 1

beta<- 1

$b<-1 ; c<-0.5$;

bigN $<-14$ 
In this model, a predator begins with prior knowledge concerning the likely palatability of each unfamiliar prey, and will consequently update its beliefs by sampling the prey and gaining evidence which shapes its posterior beliefs. The information a predator has gathered is represented by its current knowledge state $(r, n)$, where $\mathrm{n}$ represents the total number of prey a predator has attacked, and $\mathrm{r}$ represents the number of prey attacked that turned out to be unpalatable. Moving forward from their current knowledge state, a predator has the choice to attack (1) or not attack future prey (0). This behavioural choice will consequently result in a unique return to the predator.

Here, the goal is to determine the maximum benefits return, $S_{\max }(r, n)$, from all remaining trials $(N-n)$ and for any given knowledge state $S(r, n)$.

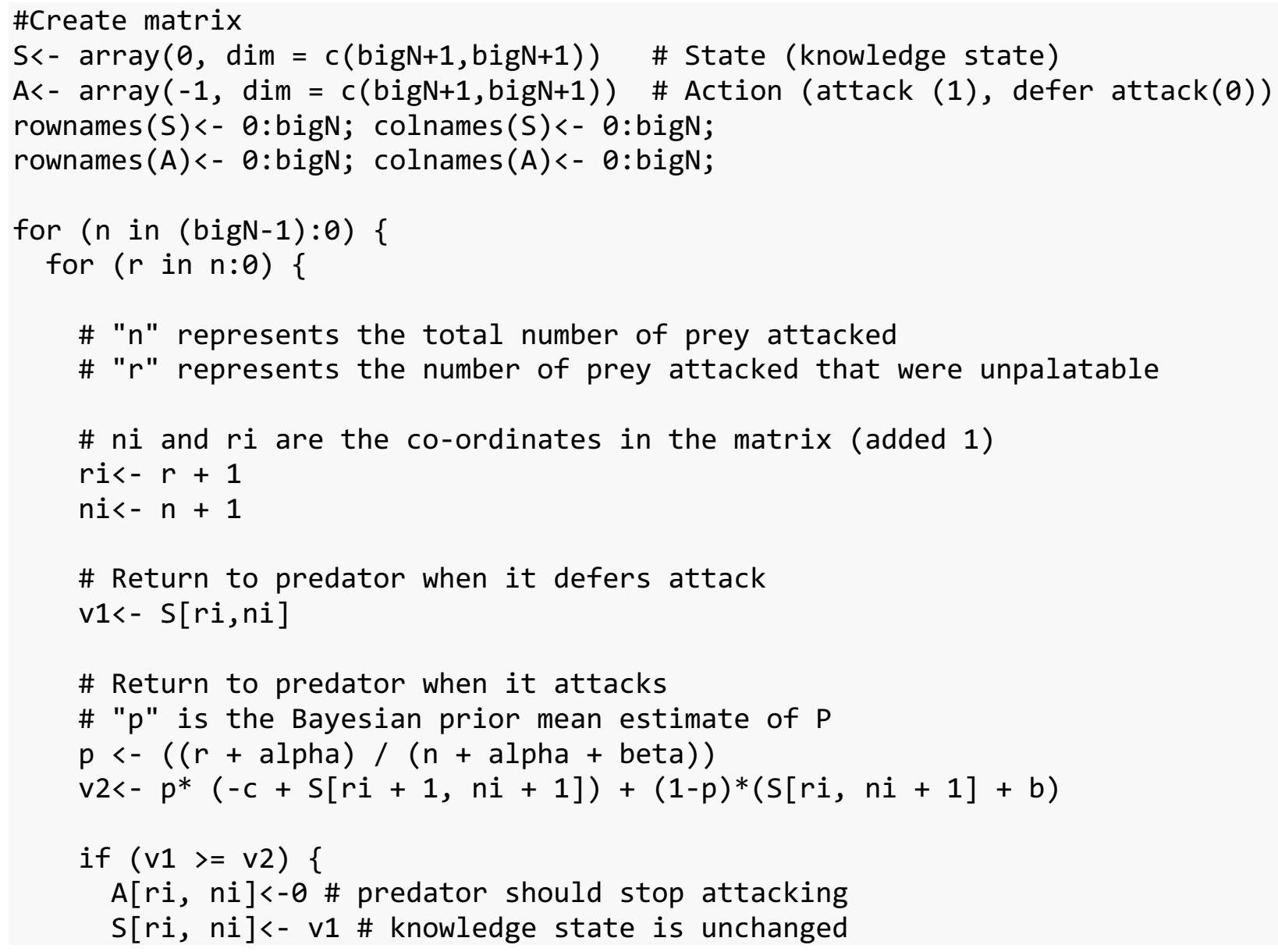




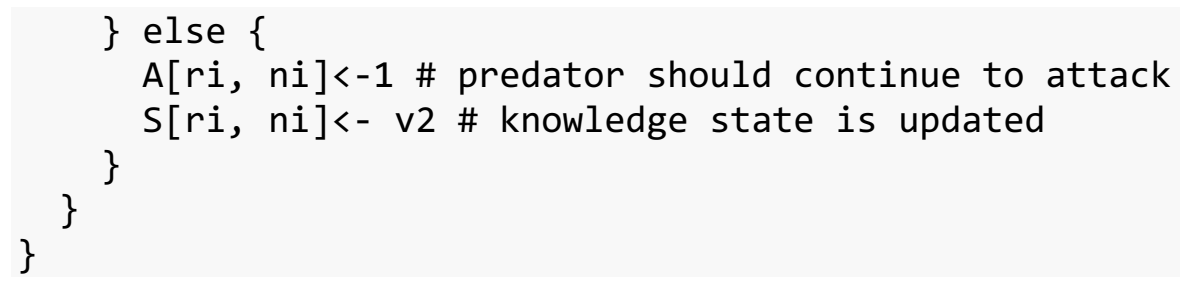

Now, we can determine the critical number of unpalatable prey a predator should consume before complete rejection.

\# Determine critical number of unpalatable prey predator should attack before complete rejection $\operatorname{subA}<-\operatorname{diag}(A)$

crit<- $\min ($ which $(\operatorname{subA}==0))-1$

\section{Simulation of a predator responding to a sequence of binary food choices}

Here, we simulate a predator adopting the optimal decision rules, as they encounter 14 binary food choices consisting of a familiar palatable prey, and an unfamiliar prey.

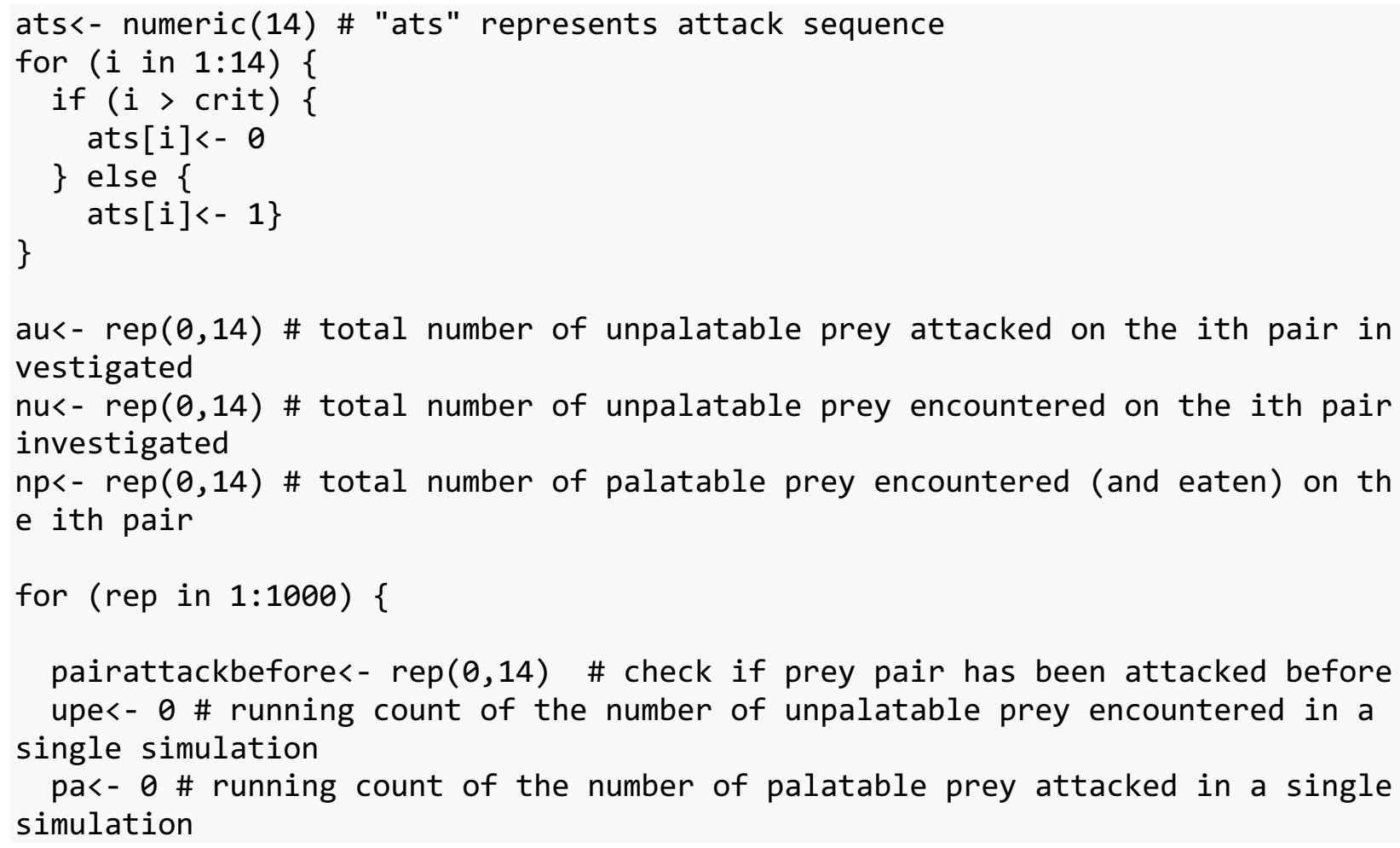


ua<- $\theta$ \# running count of the number of unpalatable prey attacked in a sing le simulation

for (i in $1: 14$ ) \{

preypair<- sample $(1: 14,1)$

while (pairattackbefore[preypair] ==1) $\{$ preypair $<-\operatorname{sample}(1: 14,1)\}$

\# this finds a prey pair that has not been sampled before

ta<- pa + ua \# total number of prey attacked

if $($ pref $==1)\{q v<-(($ alpha + ua $) /($ alpha+beta+ta $))\}$

\# if a predator shows a preference, it chooses the rewarding prey type ba sed from its prior expectation

if (pref ==0) $\{q v<-0.5\}$

\# if a predator does not show a preference, it chooses the rewarding prey type at random (50\%)

if (runif(1)< qv) \{

$n p[i]<-n p[i]+1$

$p a<-p a+1$

pairattackbefore[preypair]<- 1

\} else \{

$\mathrm{nu}[\mathrm{i}]<-\mathrm{nu}[\mathrm{i}]+1$

upe $<-$ upe +1

if (ats[upe] == 1) \{

$\mathrm{au}[\mathrm{i}]<-\mathrm{au}[\mathrm{i}]+1$

ua<- ua +1

pairattackbefore[preypair $]<-1\}$

\}

\} 
To visualize these results, we can plot the probability that the prey attacked is palatable against the total number of pairs the predator encounters.

plot $(1: 14, \mathrm{np}[1: 14] /(\mathrm{np}[1: 14]+\mathrm{au}[1: 14])$, type $=$ "o", pch $=20$, main=expression (paste(alpha,"=1,", beta, "=1")), font.main=1, cex.main=1, $x l a b=" N u m b e r$ of pairs encountered", ylab="Probability that the prey atta cked is palatable")

$$
\alpha=1, \beta=1
$$

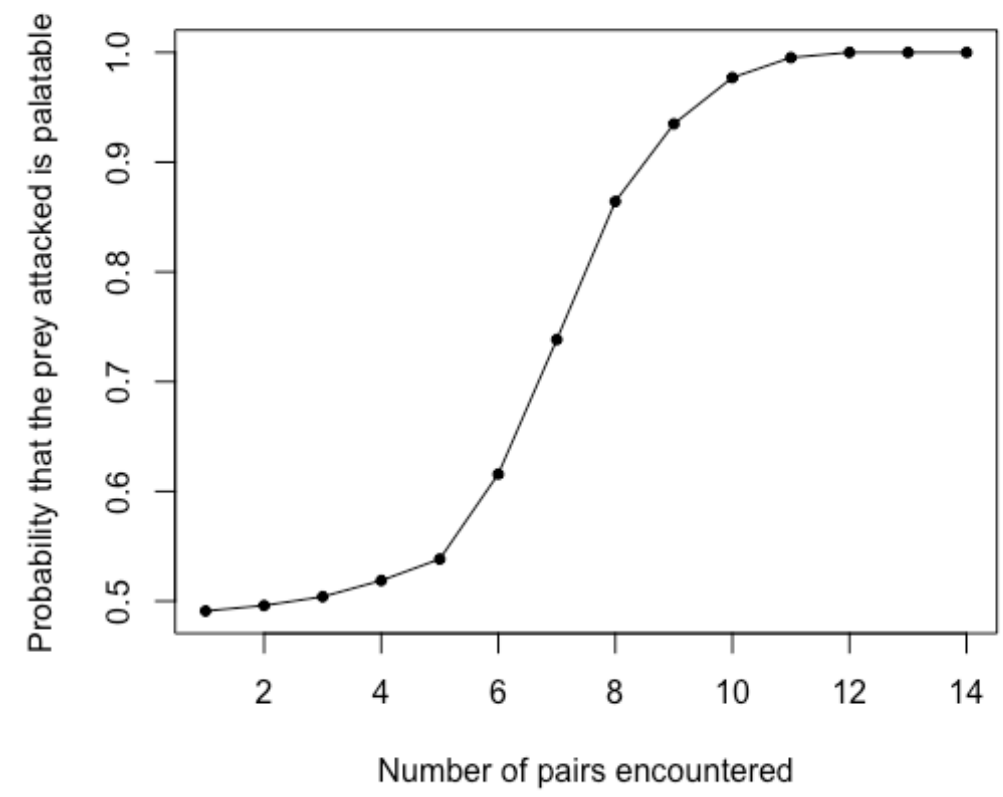

Figure A1. Optimal sampling strategy of a predator faced with a sequence of 14 binary food choices consisting of a familiar (palatable) and unfamiliar prey type. The predator begins with an expectation of $0.5(\alpha=1$ and $\beta=1)$ that unfamiliar prey are palatable. I assumed that the predator uses Bayesian learning in making prey-choice decisions. 